\title{
Smoothness of Orlicz function spaces equipped with the $p$-Amemiya norm
}

\section{Xiaoyan $\mathrm{Li}^{1,2} \cdot$ Yunan $\mathrm{Cui}^{1} \cdot$ Marek Wisla $^{3}$}

Received: 5 January 2021 / Accepted: 17 April 2021 / Published online: 7 May 2021

(c) The Author(s) 2021

\begin{abstract}
In this paper, we will use the convex modular $\rho^{*}(f)$ to investigate $\|f\|_{\Psi, q}^{*}$ on $\left(L_{\Phi}\right)^{*}$ defined by the formula $\|f\|_{\Psi, q}^{*}=\inf _{k>0} \frac{1}{k} s_{q}\left(\rho^{*}(k f)\right)$, which is the norm formula in Orlicz dual spaces equipped with $p$-Amemiya norm. The attainable points of dual norm $\|f\|_{\Psi, q}^{*}$ are discussed, the interval for dual norm $\|f\|_{\Psi, q}^{*}$ attainability is described. By presenting the explicit form of supporting functional, we get sufficient and necessary conditions for smooth points. As a result, criteria for smoothness of $L_{\Phi, p}(1 \leq p \leq \infty)$ is also obtained. The obtained results unify, complete and extended as well the results presented by a number of paper devoted to studying the smoothness of Orlicz spaces endowed with the Luxemburg norm and the Orlicz norm separately.
\end{abstract}

Keywords Orlicz space $\cdot p$-Amemiya norm $\cdot$ Supporting functional $\cdot$ Smooth point Mathematics Subject Classification 46E30 - 46E20 - 46B45 - 46B42 · 46A80

Communicated by Thomas Schlumprecht.

Yunan Cui

cuiya@hrbust.edu.cn

Xiaoyan Li

li-xiaoyan0023@163.com

Marek Wisla

mwisla@amu.edu.pl

1 Department of Mathematics, Harbin University of Science and Technology, Harbin 150080, PR China

2 Department of Mathematics, Harbin Normal University, Harbin 150025, PR China

3 Faculty of Mathematics and Computer Science, Adam Mickiewicz University, ul. Umultowska 87, 61-614 Poznan, Poland 


\section{Introduction}

It is well known that smooth points and smoothness are basic concepts in geometric theory of Banach spaces. Smoothness of Orlicz spaces are of importance in applications of the approximation theory, the conditional expectation theory, probability limit theorems and the nonlinear prediction theory as well as in other applications. Criteria for smooth points and smoothness of Orlicz function and sequence spaces equipped with Luxemburg norm were given in $[4,13,28]$. Criteria for smooth points and smoothness of Orlicz function and sequence spaces equipped with Orlicz norm were given in $[5,7,26]$. But up to now, the smoothness of Orlicz function spaces equipped with $p$-Amemiya norm has not been solved. The aim of this paper is to present criteria for smooth points and smoothness of Orlicz function spaces equipped with the p-Amemiya norm.

The rest of the paper is organized as follows. In the first part of the paper some basic notions, terminology and original results are reviewed, which will be used throughout the paper. We also recalled some properties of outer function which were introduced by Wisla in [30] and Köthe predual, i.e., $\left(E_{\Phi, p}\right)^{*}=L_{\Psi, q}$ where $\frac{1}{p}+\frac{1}{q}=1$ and $\Psi$ is the function complementary to the Orlicz function $\Phi$ in the sense of Young. In the next part of the paper, we will use the convex modular $\rho^{*}(f)$ to investigate $\|f\|_{\Psi, q}^{*}$ on $\left(L_{\Phi}\right)^{*}$ defined by the formula $\|f\|_{\Psi, q}^{*}=\inf _{k>0} \frac{1}{k} s_{q}\left(\rho^{*}(k f)\right)$, which is the norm formula in Orlicz dual spaces equipped with $p$-Amemiya norm. The attainable points of dual norm $\|f\|_{\Psi, q}^{*}$ are discussed, the interval for dual norm $\|f\|_{\Psi, q}^{*}$ attainability is described. In the last part of the paper, we present the explicit form of supporting functional and get sufficient and necessary conditions for smooth points. As a result, criteria for smoothness of $L_{\Phi, p}(1 \leq p \leq \infty)$ are obtained.

Let $X$ be a real Banach space, and $S(X)$ be the unit sphere of $X$. By $X^{*}$ we denote the dual space of $X$. In the sequel $N$ and $R$ denote the set of natural numbers and the set of real numbers, respectively.

For any map $\Phi: R \rightarrow[0, \infty]$ define

$$
a_{\Phi}=\sup \{u \geq 0: \Phi(u)=0\}, \quad b_{\Phi}=\sup \{u>0: \Phi(u)<\infty\} .
$$

Notice that if $\Phi$ is even on $R, a_{\Phi}=0$ means that $\Phi$ vanishes only at zero while $b_{\Phi}=\infty$ means that $\Phi$ takes only finite values.

A map $\Phi: R \rightarrow[0, \infty]$ is said to be an Orlicz function if $\Phi(0)=0, \Phi$ is not identically equal to zero (i.e., $\lim _{u \rightarrow \infty} \Phi(u)=\infty$ ), $\Phi$ is even and convex on the interval $\left(-b_{\Phi}, b_{\Phi}\right)$ and left-continuous at $b_{\Phi}$ i.e., $\lim _{u \rightarrow b_{\Phi}^{-}} \Phi(u)=\Phi\left(b_{\Phi}\right)$. Let us notice that every Orlicz function $\Phi$ is continuous on the interval $\left(-b_{\Phi}, b_{\Phi}\right)$. Recall also that an Orlicz function $\Phi$ is called an $\mathrm{N}$-function if it vanishes only at 0 , takes only finite values and the following two conditions are satisfied: $\lim _{u \rightarrow 0} \frac{\Phi(u)}{u}=0$ and $\lim _{u \rightarrow \infty} \frac{\Phi(u)}{u}=\infty$.

For every Orlicz function $\Phi$, we define its complementary function (in the sense of Young) $\Psi: R \rightarrow[0, \infty]$ by the formula

$$
\Psi(v)=\sup \{u|v|-\Phi(u): u \geq 0\} .
$$


It is well known that the complementary function $\Psi$ is also an Orlicz function whenever $\frac{\Phi(u)}{u} \rightarrow 0$ as $u \rightarrow 0$ (see [18] ).

In the following, by $p_{+}(u)$ and $p_{-}(u)\left(q_{+}(v)\right.$ and $\left.q_{-}(v)\right)$ we will denote the right and left derivatives of $\Phi(u)(\Psi(v))$ at $u(v)$ respectively. Here we define $p_{+}\left(b_{\Phi}\right)=\infty$ and $p_{-}(u)=\infty$ for all $u>b_{\Phi}\left(q_{+}\left(b_{\Psi}\right)=\infty\right.$ and $q_{-}(v)=\infty$ for all $\left.v>b_{\Psi}\right)$.

For every $u, v \in R$, we have the following Young Inequality:

$$
|u v| \leq \Phi(u)+\Psi(v)
$$

which reduces to an equality when $v \in\left[p_{-}(u), p_{+}(u)\right]$ if $u$ is given, or when $u \in\left[q_{-}(v), q_{+}(v)\right]$ if $v$ is given (see [6]).

Let us underline that $p_{+}, p_{-}, q_{+}, q_{-}$will always mean functions, while letters $p, q$ will always refer to numbers.

Let $(G, \Sigma, \mu)$ be a measure space with a $\sigma$-finite, nonatomic and complete measure $\mu$ and $L^{0}(\mu)$ be the set of all $\mu$-equivalence classes of real and $\Sigma$-measurable functions defined on $G$. To simplify notations, by a characteristic function $\chi_{A}$ of a subset $A \subset G$ we will mean the function defined by

$$
\chi_{A}(t)= \begin{cases}1, & \text { for } t \in A, \\ 0, & \text { for } t \notin A .\end{cases}
$$

For a given Orlicz function $\Phi$ we define on $L^{0}(\mu)$ a convex functional (called a pseudomodular [21]) by

$$
I_{\Phi}(x)=\int_{G} \Phi(x(t)) \mathrm{d} \mu .
$$

The Orlicz space $L_{\Phi}$ generated by an Orlicz function $\Phi$ is a linear space of measurable functions defined by the formula

$$
L_{\Phi}=\left\{x \in L^{0}(\mu): I_{\Phi}(c x)<\infty \text {, for some } c>0 \text { depending on } x\right\} .
$$

By $E_{\Phi}$ we denote the linear space of all measurable functions such that $I_{\Phi}(c x)<\infty$ for all $c>0$. It may happen that the space $E_{\Phi}$ consists of only one element-the zero function. For instance, this happens if the measure $\mu$ is atomless and the function $\Phi$ jumps to infinity (i.e., $b_{\Phi}<\infty$ ).

The Orlicz space $L_{\Phi}$ is a Banach space when it is endowed with any of the norms:

$$
\begin{aligned}
& \|u\|_{\Phi}=\inf \left\{\varepsilon>0: I_{\Phi}(u / \varepsilon) \leq 1\right\} \\
& \|u\|_{\Phi}^{\circ}=\sup \left\{\int_{G}|u(t) v(t)| \mathrm{d} \mu: v \in L_{\Psi}, I_{\Psi}(v) \leq 1\right\}
\end{aligned}
$$

and

$$
\|u\|_{\Phi}^{A}=\inf _{k>0} \frac{1}{k}\left(1+I_{\Phi}(k u)\right)
$$

which are called the Luxemburg norm, Orlicz norm and Amemiya norm, respectively. Krasnoslskii and Rutickii [18], Nakano [23], Luxemburg and Zaanen [20] 
proved, under additional assumptions on the function $\Phi$, that the Orlicz norm can be expressed exactly by the Amemiya formula, i.e., $\|x\|_{\Phi}^{\circ}=\|x\|_{\Phi}^{A}$. In the most general case of Orlicz function $\Phi$, the similar result was obtained by Hudzik and Maligranda ([15]). Moreover, it is not difficult to verify that Luxemburg norm can also be expressed by an Amemiya-like formula (see [9, 24]), namely

$$
\|u\|_{\Phi}=\inf _{k>0} \frac{1}{k} \max \left\{1, I_{\Phi}(k u)\right\} .
$$

In the paper [15], Hudzik and Maligranda proposed to investigate another class of norms given by the Amemiya formula-norms generated by the functions of the type

$$
s_{p}(u)= \begin{cases}\left(1+u^{p}\right)^{\frac{1}{p}}, & \text { for } 1 \leq p<\infty, \\ \max \{1, u\}, & \text { for } p=\infty,\end{cases}
$$

and

$$
\|u\|_{\Phi, p}=\inf _{k>0} \frac{1}{k} s_{p}\left(I_{\Phi}(k u)\right) \quad(1 \leq p \leq \infty)
$$

In that case we obtain a family of topologically equivalent norms (called $p$-Amemiya norms and denoted by $\|\cdot\|_{\Phi, p}$, indexed by $1 \leq p \leq \infty$ and satisfying the inequalities

$$
\|u\|_{\Phi}=\|u\|_{\Phi, \infty} \leq\|u\|_{\Phi, p} \leq\|u\|_{\Phi, q} \leq\|u\|_{\Phi, 1}=\|u\|_{\Phi}^{o} \leq 2\|u\|_{\Phi}
$$

for all $1 \leq q \leq p \leq \infty$.

Since that time, an intensive development of research connected with Orlicz spaces equipped with $p$-Amemiya norms have taken place, many important results broaden the knowledge about the geometry of these spaces (see $[3,8,9$, $11,12,14,17,19])$ and some open questions were put (see [29]).

To simplify notation, the Orlicz spaces equipped with the $p$-Amemiya norms are denoted by $L_{\Phi, p}=\left(L_{\Phi},\|\cdot\|_{\Phi, p}\right)$. Further, for any function $u \in L^{0}$ the essential supremum of $|u|$ over $G$, i.e. supees $e_{t \in G}|u(t)|$, no matter whether this number is finite or not, will be denoted by $\|u\|_{\infty}$.

We say an Orlicz function $\Phi$ satisfies the $\Delta_{2}$-condition for all $u \in R$ (resp., at infinity) [resp., at zero] if there is a constant $K>0$ (resp., and a constant $u_{0} \geq 0$ with $\left.\Phi\left(u_{0}\right)<\infty\right)$ [resp., and a constant $u_{0}>0$ with $\Phi\left(u_{0}\right)>0$ ] such that $\Phi(2 u) \leq K \Phi(u)$ for all $u \in R$ (resp., for every $|u| \geq u_{0}$ ) [resp., for every $|u| \leq u_{0}$ ). We will shortly write $\Phi \in \Delta_{2}(R)$ (resp., $\Phi \in \Delta_{2}(\infty)$ ) [resp., $\Phi \in \Delta_{2}(0)$ ]. Evidently, $\Phi \in \Delta_{2}(R)$ if and only if $\Phi \in \Delta_{2}(\infty)$ and $\Phi \in \Delta_{2}(0)$.

We say that an Orlicz function $\Phi$ satisfies the suitable $\Delta_{2}(\mu)$-condition if $\Phi \in \Delta_{2}(0)$ provided $\mu$ is purely atomic, $\Phi \in \Delta_{2}(\infty)$ provided $\mu$ is non-atomic and $\mu(G)<\infty$ and $\Phi \in \Delta_{2}(R)$ in the case of $\mu(G)=\infty$.

Further details about Orlicz spaces equipped with the Luxemburg or the Orlicz norm, can be found in $[2,6,18,20-22,24,25,31]$. Basic results on the Orlicz spaces equipped with $p$-Amemiya norms have been presented in [9]. 


\section{Auxiliary result}

In the paper [9], Cui et al. introduced the function $\alpha_{p}: L_{\Phi, p} \rightarrow[-1, \infty]$ by

$$
\alpha_{p}(u)= \begin{cases}I_{\Phi}^{p-1}(u) I_{\Psi}\left(p_{+}(|u|)\right)-1, & \text { for } 1 \leq p<\infty \\ -1, & \text { for } p=\infty, I_{\Phi}(u) \leq 1, \\ I_{\Psi}\left(p_{+}(|u|)\right), & \text { for } p=\infty, I_{\Phi}(u)>1 .\end{cases}
$$

and the functions $k_{p}^{*}: L_{\Phi, p} \rightarrow[0, \infty), k_{p}^{* *}: L_{\Phi, p} \rightarrow(0, \infty]$ by $k_{p}^{*}(u)=\inf \{k \geq 0:$ $\left.\alpha_{p}(k u) \geq 0\right\}$ ( with $\left.\inf \emptyset=\infty\right), k_{p}^{* *}(u)=\sup \left\{k \geq 0: \alpha_{p}(k u) \leq 0\right\}$.

It is evident that $k_{p}^{*}(u) \leq k_{p}^{* *}(u)$ for every $1 \leq p \leq \infty$ and $u \in L_{\Phi, p} \backslash\{0\}$.

Set $K_{p}(u)=\left\{0<k<\infty: k_{p}^{*}(u) \leq k \leq k_{p}^{* *}(u)\right\}$.

Lemma 2.1 [9] For every $1 \leq p \leq \infty$ and $u \in L_{\Phi, p} \backslash\{0\}$, the following conditions hold:

(i) If $k_{p}^{*}(u)=k_{p}^{* *}(u)=\infty, K_{p}(u)=\emptyset$, then $\|u\|_{\Phi, p}=\lim _{k \rightarrow \infty} \frac{1}{k}\left(1+I_{\Phi}^{p}(k u)\right)^{\frac{1}{p}}$.

(ii) If $k_{p}^{*}(u)<k_{p}^{* *}(u)=\infty$, then the $p$-Amemiya norm $\|u\|_{\Phi, p}$ is attained at every $k \in\left[k_{p}^{*}(u), \infty\right)$.

(iii) If $k_{p}^{* *}(u)<\infty$, then the $p$-Amemiya norm $\|u\|_{\Phi, p}$ is attained at every $k \in\left[k_{p}^{*}(u), k_{p}^{* *}(u)\right]$.

Lemma 2.2 [9] Let $\Phi$ be an Orlicz function and let $1 \leq p \leq \infty$. The set $K_{p}(u)$ is nonempty if and only if one of the following conditions is satisfied:

(i) If $p=1$ then $\Phi$ does not admit an asymptote at infinity.

(ii) If $1<p<\infty$ then $\Phi$ is not linear on $[0, \infty)$.

(iii) If $p=\infty$, then for every Orlicz function $\Phi$ is $K_{p}(u) \neq \emptyset$.

(iv) $\Phi$ takes infinite values.

Remark 2.3 By Lemma 2.2, we know for every $1 \leq p<\infty$, if $K_{p}(u)=\emptyset$, then there exists $G_{0} \subset G$ such that $L_{\Phi}\left(G_{0}\right)$ is linearly isometric to $L_{1}$. We know that $L_{\infty}$ is the dual space of $L_{1}$ and $L_{1}$ is not a smooth space. For this reason we will assume $K_{p}(u) \neq \emptyset$ in the following whenever smooth points and smoothness are considered.

The $p$-Amemiya norm is defined by using of two functions: the (inner) Orlicz function $\Phi$ (more precisely: the modular $I_{\Phi}$ ) and the outer function $s_{p}$ defined on the half line $[0, \infty)$ by

$$
s_{p}(u)= \begin{cases}\left(1+u^{p}\right)^{\frac{1}{p}}, & \text { for } 1 \leq p<\infty, \\ \max \{1, u\}, & \text { for } p=\infty\end{cases}
$$

The family $\left\{s_{p}(\cdot): 1 \leq p \leq \infty\right\}$ consists of convex, nondecreasing on $[0, \infty)$ functions with exactly one common point (knot) at 0 (i.e., $s_{p}(0)=1$ for all $1 \leq p \leq \infty$ ). Moreover, on the half-line $[0, \infty)$, the functions $s_{p}(\cdot)$ are strictly increasing for 
$1 \leq p<\infty$, strictly convex for $1<p<\infty$, and $s_{p}(u)<s_{q}(u)$ for every $1 \leq q<p \leq \infty$ and $u>0$.

In the paper [30], Wisla introduced outer functions and presented basic properties of outer functions. We recall them here. A function $s:[0, \infty) \rightarrow[1, \infty)$ will be called an outer function, if it is convex and

$$
\max \{1, u\} \leq s(u) \leq 1+u \text { for all } u \geq 0 .
$$

To simplify notations, we extend the domain and range of $s$ to the interval $[0, \infty]$ by setting $s(\infty)=\infty$.

Evidently, for every $1 \leq p \leq \infty, s_{p}(\cdot)$ is an outer function. We will say that two outer functions $s, \sigma$ are conjugate (to each other) in the Hölder sense, if $u+v \leq s(u) \sigma(v)$ for all $u, v \geq 0$.

Lemma 2.4 [30] The outer function $\sigma(v)=1+v$ is conjugate in the Hölder sense to any outer function $s(\cdot)$.

Lemma 2.5 [30] For any outer function $s(\cdot)$ the function $s^{*}(\cdot)$ defined by $s^{*}(v)=\sup _{u \geq 0} \frac{u+v}{s(u)}, 0 \leq v<\infty, s^{*}(\infty)=\infty$, is the minimal outer function conjugate to $s(\cdot)$ in the Hölder sense.

Lemma 2.6 [30] If $s_{p}(u)=\left(1+u^{p}\right)^{\frac{1}{p}}$ then $s_{p}^{*}(v)=s_{q}(v)=\left(1+v^{q}\right)^{\frac{1}{q}}$ for all $1<p, q<\infty \quad$ with $\frac{1}{p_{1}}+\frac{1}{q}=1$. And the Hölder equality $u+v=s_{p}(u)_{1} \cdot s_{q}(v)=\left(1+u^{p}\right)^{\frac{1}{p}} \cdot\left(1+v^{q}\right)^{\frac{1}{q}}$ for all $0<u, v<\infty$ holds true if and only if $u^{q} \cdot v^{\underline{p}}=1$ (i.e., $u^{p-1} \cdot v=1$ or $\left.u \cdot v^{q-1}=1\right)$.

Lemma 2.7 [30] Let $\Phi, \Psi$ be the Orlicz functions complementary in the sense of Young that take finite values only. If the $p$-Amemiya norm $\|\cdot\|_{\Phi, p}$ is $k_{p}^{*}$-finite $(1 \leq p \leq \infty)$ then $\left(E_{\Phi},\|\cdot\|_{\Phi, p}\right)$ is the Köthe predual of the Orlicz space $\left(L_{\Psi},\|\cdot\|_{\Psi, q}\right)$, i.e., $\left(E_{\Phi, p}\right)^{*}=L_{\Psi, q}$.

Orlicz spaces are endowed with the structure of Banach lattices [1]. This property can be used in a more refined analysis of the (topological) dual space of $L_{\Phi}$, which is denoted by $\left(L_{\Phi}\right)^{*}$. $\left(L_{\Phi}\right)^{*}$ is represented in the following way (see [21]): $\left(L_{\Phi}\right)^{*}=L_{\Psi} \oplus F$, i.e, every $f \in\left(L_{\Phi, p}\right)^{*}(1 \leq p \leq \infty)$ is a uniquely represented in the form

$$
f=v+\varphi,
$$

where $\varphi$ is singular functional, i.e., $\varphi(u)=0$ for any $u \in E_{\Phi, p}$ and $v \in L_{\Psi, q}$ where $\frac{1}{p}+\frac{1}{q}=1$ and $\Psi$ is the function complementary to the Orlicz function $\Phi$ in the sense of Young, is the regular functional by the formula:

$$
u(v)=\int_{G} u(t) v(t) \mathrm{d} t, \quad \text { for all } u \in L_{\Phi, p} .
$$

Let us define for each $f \in\left(L_{\Phi}\right)^{*}$ : 


$$
\|f\|_{\Psi}^{o}=\sup \left\{f(u):\|u\|_{\Phi}=1\right\}, \quad\|f\|_{\Psi}=\sup \left\{f(u):\|u\|_{\Phi}^{o}=1\right\} .
$$

Proofs of the next three lemmas can be found for N-functions $\Phi$ in [16], but they are also true for arbitrary Orlicz functions $\Phi$ (see [27], even in the more general case of Musielak-Orlicz functions).

Lemma 2.8 [27] Let $f \in\left(L_{\Phi}\right)^{*}$ be as in (2). Then $\|f\|_{\Psi}^{o}=\|v\|_{\Psi}^{o}+\|\varphi\|^{o}$.

Lemma 2.9 [27] For any $\varphi \in F$,

$$
\|\varphi\|=\|\varphi\|^{o}=\sup \left\{\varphi(u): I_{\Phi}(u)<\infty\right\}=\sup \left\{\frac{\varphi(u)}{\theta(u)}: u \in L_{\Phi} \backslash E_{\Phi}\right\},
$$

where $\theta(u)=\inf \left\{\lambda>0, I_{\Phi}\left(\frac{u}{\lambda}\right)<\infty\right\}$.

Lemma 2.10 [27] If $f \in\left(L_{\Phi}\right)^{*}$ is of the form (2), then

$$
\|f\|_{\Psi}=\inf \left\{\lambda>0: I_{\Psi}\left(\frac{v}{\lambda}\right)+\frac{\|\varphi\|}{\lambda} \leq 1\right\} .
$$

\section{The dual norm $\|\cdot\|_{\Psi, q}^{*}$ and norm attainability}

Let $f \in\left(L_{\phi}\right)^{*}$ be as in (2). Define

$$
\rho^{*}(f)=I_{\Psi}(v)+\|\varphi\| .
$$

Cui et al. proved that $\rho^{*}(f)$ is a convex modular in $\left(L_{\Phi}\right)^{*}$ (see [10]). Now, for $1 \leq p \leq \infty$, on $\left(L_{\Phi, p}\right)^{*}$ we introduce new functionals as follows

$$
\|f\|_{\Psi, q}^{*}= \begin{cases}\inf _{k>0} \frac{1}{k}\left(1+\left(\rho^{*}(k f)\right)^{q}\right)^{\frac{1}{q}}=\inf _{k>0} \frac{1}{k} s_{q}\left(\rho^{*}(k f)\right), & \text { for } 1 \leq q<\infty, \\ \inf _{k>0} \frac{1}{k} \max \left\{1, \rho^{*}(k f)\right\}, & \text { for } q=\infty,\end{cases}
$$

where $f=v+\varphi$ is of the form (2). Evidently, $\|f\|_{\Psi, 1}^{*}=\|f\|_{\Psi}^{o}$. In the next section we will prove that $\|f\|_{\Psi}=\|f\|_{\Psi, \infty}^{*}$. We will also prove there that for any $1 \leq q \leq \infty$ the functional $\|f\|_{\Psi, q}^{*}$ is a norm on $\left(L_{\Phi, p}\right)^{*}$ and all the norms $\|f\|_{\Psi, q}^{*}$ are equivalent to each other.

Theorem 3.1 The $\|f\|_{\Psi}$ and $\|f\|_{\Psi, \infty}^{*}$ coincide, i.e.,

$$
\|f\|_{\Psi}=\|f\|_{\Psi, \infty}^{*}=\inf _{k>0} \frac{1}{k} \max \left\{1, \rho^{*}(k f)\right\}, \quad \text { for all } f \in\left(L_{\Phi, 1}\right)^{*} .
$$

Proof For any $f \in\left(L_{\Phi, 1}\right)^{*}, \rho^{*}(f)>1$ implies $\rho^{*}(f) \geq\|f\|_{\Psi}$. If there exists $f \in\left(L_{\Phi, 1}\right)^{*}$, with $\rho^{*}(f)>1$ and $1<\rho^{*}(f)<\|f\|_{\Psi}$, we have 


$$
1<\rho^{*}\left(\frac{f}{\rho^{*}(f)}\right) \leq \frac{1}{\rho^{*}(f)} \rho^{*}(f)=1,
$$

a contradiction. Thus, $\rho^{*}\left(\frac{f}{\lambda}\right)>1$ implies $\lambda \rho^{*}\left(\frac{f}{\lambda}\right) \geq\|f\|_{\Psi}$, so

$$
\begin{aligned}
\|f\|_{\Psi} & =\inf _{\rho^{*}\left(\frac{f}{\lambda}\right) \leq 1} \lambda=\min \left\{\inf _{\rho^{*}\left(\frac{f}{\lambda}\right) \leq 1} \lambda, \inf _{\rho^{*}\left(\frac{f}{\lambda}\right)>1} \lambda \rho^{*}\left(\frac{f}{\lambda}\right)\right\} \\
& =\min \left\{\inf _{\rho^{*}(k f) \leq 1} \frac{1}{k}, \inf _{\rho^{*}(k f)>1} \frac{1}{k} \rho^{*}(k f)\right\} \\
& =\inf _{k>0} \frac{1}{k} \max \left\{1, \rho^{*}(k f)\right\}=\|f\|_{\Psi, \infty}^{*} .
\end{aligned}
$$

Theorem 3.2 Let $\rho^{*}(f)$ be as in (3). The functional

$$
\|f\|_{\Psi, q}^{*}=\inf _{k>0} \frac{1}{k}\left(1+\left(\rho^{*}(k f)\right)^{q}\right)^{\frac{1}{q}}=\inf _{k>0} \frac{1}{k} s_{q}\left(\rho^{*}(k f)\right) \quad(1 \leq q \leq \infty)
$$

is a norm on $\left(L_{\Phi, p}\right)^{*}$ where $\frac{1}{p}+\frac{1}{q}=1$ which is equivalent to $\|f\|_{\Psi}$ :

$$
\|f\|_{\Psi} \leq\|f\|_{\Psi, q}^{*} \leq 2^{\frac{1}{q}}\|f\|_{\Psi} .
$$

Proof In the case $q=\infty$ the thesis follows directly from Theorem 3.1. So, we can assume that $1 \leq q<\infty$.

Let $\lambda \in R$. Then

$$
\|\lambda f\|_{\Psi, q}^{*}=\inf _{k>0} \frac{1}{k} s_{q}\left(\rho^{*}(k \lambda f)\right)=|\lambda| \inf _{k>0} \frac{1}{k|\lambda|} s_{q}\left(\rho^{*}(k \lambda f)\right)=|\lambda| \cdot\|f\|_{\Psi, q}^{*},
$$

so $\|\cdot\|_{\Psi, q}^{*}$ is homogeneous.

Let $f_{1}, f_{2} \in\left(L_{\Phi, p}\right)^{*} \backslash\{0\}$ and $\varepsilon>0$. We can find $k, l>0$ such that $\frac{1}{k} s_{q}\left(\rho^{*}\left(k f_{1}\right)\right) \leq\left\|f_{1}\right\|_{\Psi, q}^{*}+\varepsilon, \frac{1}{l} s_{q}\left(\rho^{*}\left(l f_{2}\right)\right) \leq\left\|f_{2}\right\|_{\Psi, q}^{*}+\varepsilon$. By the convexity of $\Psi$ and $s_{q}$, we have

$$
\begin{aligned}
\left\|f_{1}+f_{2}\right\|_{\Psi, q}^{*} & \leq \frac{k+l}{k l} s_{q}\left(\rho^{*}\left(\frac{k l}{k+l}\left(f_{1}+f_{2}\right)\right)\right) \\
& =\frac{k+l}{k l} s_{q}\left(\rho^{*}\left(\frac{l}{k+l} k f_{1}+\frac{k}{k+l} l f_{2}\right)\right) \\
& \leq \frac{k+l}{k l} s_{q}\left(\frac{l}{k+l} \rho^{*}\left(k f_{1}\right)+\frac{k}{k+l} \rho^{*}\left(l f_{2}\right)\right) \\
& \leq \frac{1}{k} s_{q}\left(\rho^{*}\left(k f_{1}\right)\right)+\frac{1}{l} s_{q}\left(\rho^{*}\left(l f_{2}\right)\right. \\
& \leq\left\|f_{1}\right\|_{\Psi, q}^{*}+\left\|f_{2}\right\|_{\Psi, q}^{*}+2 \varepsilon .
\end{aligned}
$$

Letting $\varepsilon \rightarrow 0$, we get the triangle inequality.

Further, by Theorem 3.1, we have 


$$
\begin{aligned}
\|f\|_{\Psi} & =\inf _{k>0} \frac{1}{k} \max \left\{1, \rho^{*}(k f)\right\} \leq \inf _{k>0} \frac{1}{k}\left(1+\left(\rho^{*}(k f)\right)^{q}\right)^{\frac{1}{q}} \\
& =\|f\|_{\Psi, q}^{*} \leq 2^{\frac{1}{q}} \inf _{\rho^{*}(k f) \leq 1} \frac{1}{k}=2^{\frac{1}{q}}\|f\|_{\Psi} .
\end{aligned}
$$

Thus (4) holds true and $\|f\|_{\Psi, q}^{*}=0 \Leftrightarrow\|f\|_{\Psi}=0 \Leftrightarrow f=0$.

In the following by the determinant function we shall mean the function defined by $\beta_{q}:\left(L_{\Phi, p}\right)^{*} \rightarrow[-1, \infty]$,

$$
\beta_{q}(f)= \begin{cases}I_{\Phi}\left(q_{+}(|v|)\right) \cdot\left(\rho^{*}(f)\right)^{q-1}-1, & \text { for } 1 \leq q<\infty \\ -1, & \text { for } q=\infty, \rho^{*}(f) \leq 1 \\ I_{\Phi}\left(q_{+}(|v|)\right), & \text { for } q=\infty, \rho^{*}(f)>1\end{cases}
$$

Further, define

$$
\begin{aligned}
& \theta^{*}:\left(L_{\Phi, p}\right)^{*} \rightarrow[0, \infty), \theta^{*}(f)=\inf \left\{k>0: \rho^{*}\left(k^{-1} f\right)<\infty\right\}, \\
& k_{q}^{*}(f):\left(L_{\Phi, p}\right)^{*} \rightarrow[0, \infty), k_{q}^{*}(f)=\inf \left\{k \geq 0: \beta_{q}(k f) \geq 0\right\} \quad(\text { with } \inf \emptyset=\infty), \\
& k_{q}^{* *}(f):\left(L_{\Phi, p}\right)^{*} \rightarrow(0, \infty], k_{q}^{* *}(f)=\sup \left\{k \geq 0: \beta_{q}(k f) \leq 0\right\} .
\end{aligned}
$$

The support of a measurable function $v \in L_{\Psi, q}$ is defined by $\operatorname{supp}(v)=\{t \in G: v(t) \neq 0\}$. In the sequel, together with a measurable function $v$, we shall often consider a sequence $\left(v_{n}\right)$ of bounded measurable functions with support of finite measure defined by

$$
v_{n}=v(t) \chi_{G_{n} \cap T_{n}},
$$

for each $n \in N, G_{n}=\{t \in G:|v(t)| \leq n\}, T_{n} \nearrow, 0<\mu\left(T_{n}\right)<\infty$ and $\bigcup_{n=1}^{\infty} T_{n}=G$.

Lemma 3.3 [9] For every $1 \leq q<\infty$ and every $a>0$

$$
\max _{x \geq 0} \frac{1+x^{q-1} a}{\left(1+x^{q}\right)^{\frac{1}{q}}}=\left(1+a^{q}\right)^{\frac{1}{q}} .
$$

Lemma 3.4 For every essentially bounded measurable function $f \in\left(L_{\Phi, p}\right)^{*}$ with support of finite measure, we have $\theta^{*}(f)=\theta_{0}^{*}(f)$ where $\theta_{0}^{*}(f)=\inf \left\{k>0, I_{\Phi}\left(q_{+}\left(\frac{|v|}{k}\right)\right)<\infty\right\}$.

Proof Suppose that $\quad\left(\theta^{*}\right)^{-1}(f)<k_{0}<\left(\theta_{0}^{*}\right)^{-1}(f)$ ( with $\inf \frac{1}{0}=\infty$ ). Then $I_{\Phi}\left(q_{+}\left(k_{0}|v|\right)\right)<\infty$, so $k_{0}\|v\|_{\infty}<b_{\Psi}$ (otherwise $I_{\Phi}\left(q_{+}(k|v|)\right)=\infty$ for every $k>k_{0}$, whence $k_{0}>\left(\theta_{0}^{*}\right)^{-1}(f)$, a contradiction). Thus

$$
I_{\Psi}\left(k_{0} v\right)+k_{0}\|\varphi\| \leq \Psi\left(k_{0}\|v\|_{\infty}\right) \cdot \mu(\operatorname{supp} v)+k_{0}\|\varphi\|<\infty .
$$

Hence $k_{0}<\left(\theta^{*}\right)^{-1}(f)$, a contradiction. 
Similarly, if $\quad\left(\theta_{0}^{*}\right)^{-1}(f)<k_{0}<\left(\theta^{*}\right)^{-1}(f)$ then $\quad I_{\Psi}\left(k_{0} v\right)+k_{0}\|\varphi\|<\infty, \quad$ so $k_{0}\|v\|_{\infty} \leq b_{\Psi}$ in this case as well. Thus

$$
I_{\Phi}\left(q_{+}\left(k_{0}|v|\right)\right) \leq \Phi\left(q_{+}\left(k_{0}\|v\|_{\infty}\right)\right) \cdot \mu(\operatorname{supp} v)<\infty .
$$

Hence $k_{0}<\left(\theta_{0}^{*}\right)^{-1}(f)$, a contradiction.

Theorem 3.5 For every $f \in\left(L_{\Phi, p}\right)^{*} \backslash\{0\}$ and every $1 \leq q<\infty$ the following conditions hold:

(i) the function $k \rightarrow \beta_{q}(k f)$ is nondecreasing on $[0, \infty)$;

(ii) $\left(0,\left(\theta^{*}\right)^{-1}(f)\right) \subset\left\{k>0: \frac{1}{k} s_{q}\left(\rho^{*}(k f)\right)<\infty\right\}$;

(iii) the function $k \rightarrow \frac{1}{k} s_{q}\left(\rho^{*}(k f)\right)$ is continuous on $\left(0,\left(\theta^{*}\right)^{-1}(f)\right)$;

(iv) the function $k \rightarrow \frac{1}{k} s_{q}\left(\rho^{*}(k f)\right)$ is decreasing on $\left(0, k_{q}^{*}(f)\right)$;

(v) the function $k \rightarrow \frac{1}{k} s_{q}\left(\rho^{*}(k f)\right)$ is nonincreasing on $\left(0, k_{q}^{* *}(f)\right)$;

(vi) the function $k \rightarrow \frac{1}{k} s_{q}\left(\rho^{*}(k f)\right)$ is increasing on $\left(k_{q}^{* *}(f),\left(\theta^{*}\right)^{-1}(f)\right)$;

(vii) the function $k \rightarrow \frac{1}{k} s_{q}\left(\rho^{*}(k f)\right)$ is nondecreasing on $\left(k_{q}^{*}(f),\left(\theta^{*}\right)^{-1}(f)\right)$.

Proof Condition (i) follows immediately from the fact that both functions $k \rightarrow I_{\Phi}\left(q_{+}(k|v|)\right)$ and $k \rightarrow \rho^{*}(k f)$ are nondecreasing on $[0, \infty)$. Condition (ii) is obvious.

(iii) The condition (iii) follows directly from the Lebesgue dominated convergence theorem.

(iv) Let $0<k_{1}<k_{2}<k_{q}^{*}(f)$ and let $f_{n}=v_{n}+\varphi, v_{n}$ be as in (5). Since, for every $n \in N$ and $0<k<k_{q}^{*}(f)$, we have

$$
I_{\Phi}\left(q_{+}\left(k\left|v_{n}\right|\right)\right) \cdot\left(\rho^{*}\left(k f_{n}\right)\right)^{q-1}=I_{\Phi}\left(q_{+}\left(k\left|v_{n}\right|\right)\right) \cdot\left(I_{\Psi}\left(k v_{n}\right)+k\|\varphi\|\right)^{q-1}<1,
$$

by Lemma 3.4, the numbers $I_{\Phi}\left(q_{+}\left(k_{i}\left|v_{n}\right|\right)\right)$ and $I_{\Psi}\left(k_{i} v_{n}\right), i=1,2$, have to be finite. Therefore

$$
\begin{aligned}
& \frac{1}{k_{2}} s_{q}\left(\rho^{*}\left(k_{2} f_{n}\right)\right)=\frac{1}{k_{2}} S_{q}\left(I_{\Psi}\left(k_{2} v_{n}\right)+k_{2}\|\varphi\|\right) \\
& =\frac{1+\left(\rho^{*}\left(k_{2} f_{n}\right)\right)^{q-1}\left(I_{\Psi}\left(k_{2} v_{n}\right)+k_{2}\|\varphi\|\right)}{k_{2}\left(1+\left(I_{\Psi}\left(k_{2} v_{n}\right)+k_{2}\|\varphi\|\right)^{q}\right)^{1-\frac{1}{q}}} \\
& =\frac{1+\left(\rho^{*}\left(k_{2} f_{n}\right)\right)^{q-1}\left(\int_{G} k_{2}\left|v_{n}(t)\right| q_{+}\left(k_{2}\left|v_{n}(t)\right|\right) \mathrm{d} t-I_{\Phi}\left(q_{+}\left(k_{2}\left|v_{n}\right|\right)\right)+k_{2}\|\varphi\|\right)}{k_{2}\left(1+\left(I_{\Psi}\left(k_{2} v_{n}\right)+k_{2}\|\varphi\|\right)^{q}\right)^{1-\frac{1}{q}}} \\
& =\frac{\left(\rho^{*}\left(k_{2} f_{n}\right)\right)^{q-1}\left(\int_{G}\left|v_{n}(t)\right| q_{+}\left(k_{2}\left|v_{n}(t)\right|\right) \mathrm{d} t+\|\varphi\|\right)-\frac{1}{k_{2}} \beta_{q}\left(k_{2} f_{n}\right)}{\left(1+\left(I_{\Psi}\left(k_{2} v_{n}\right)+k_{2}\|\varphi\|\right)^{q}\right)^{1-\frac{1}{q}}} .
\end{aligned}
$$

Let $\varepsilon_{n}=\min \left\{1,\left(\frac{1}{k_{2}}-\frac{1}{k_{1}}\right) \beta_{q}\left(k_{2} f_{n}\right)\left(1+\left(\rho^{*}\left(k_{2} f_{n}\right)\right)^{q}\right)^{\frac{1}{q}-1}\right\}$. Since $k_{2}<k_{q}^{*}(f)$, we have $\beta_{q}\left(k_{2} f_{n}\right) \leq \beta_{q}\left(k_{2} f\right)<0$, so $\varepsilon_{n}>0$. By the Young Inequality and Lemma 3.3, we obtain 


$$
\begin{aligned}
\frac{1}{k_{2}} s_{q}\left(\rho^{*}\left(k_{2} f_{n}\right)\right) & \\
\leq & \frac{\left(\rho^{*}\left(k_{2} f_{n}\right)\right)^{q-1}\left(\int_{G}\left|v_{n}(t)\right| q_{+}\left(k_{2}\left|v_{n}(t)\right|\right) \mathrm{d} t+\|\varphi\|\right)-\frac{1}{k_{1}} \beta_{q}\left(k_{2} f_{n}\right)}{\left(1+\left(\rho^{*}\left(k_{2} f_{n}\right)\right)^{q}\right)^{1-\frac{1}{q}}}-\varepsilon_{n} \\
= & \frac{1+\left(\rho^{*}\left(k_{2} f_{n}\right)\right)^{q-1}\left(\int_{G} k_{1}\left|v_{n}(t)\right| q_{+}\left(k_{2}\left|v_{n}(t)\right|\right) \mathrm{d} t+k_{1}\|\varphi\|-I_{\Phi}\left(q_{+}\left(k_{2}\left|v_{n}\right|\right)\right)\right.}{k_{1}\left(1+\left(I_{\Psi}\left(k_{2} v_{n}\right)+k_{2}\|\varphi\|\right)^{q}\right)^{1-\frac{1}{q}}} \\
\leq & \frac{1+\left(\rho_{n}^{*}\left(k_{2} f_{n}\right)\right)^{q-1}\left(I_{\Psi}\left(k_{1} v_{n}\right)+k_{1}\|\varphi\|\right)}{k_{1}\left(1+\left(I_{\Psi}\left(k_{2} v_{n}\right)+k_{2}\|\varphi\|\right)^{q}\right)^{1-\frac{1}{q}}}-\varepsilon_{n} \\
\leq & \frac{1}{k_{1}}\left(1+\left(\rho^{*}\left(k_{1} f_{n}\right)\right)^{q}\right)^{\frac{1}{q}}-\varepsilon_{n}=\frac{1}{k_{1}} s_{q}\left(\rho^{*}\left(k_{1} f_{n}\right)\right)-\varepsilon_{n} .
\end{aligned}
$$

Letting $n \rightarrow \infty$, we get $\varepsilon_{n} \rightarrow \varepsilon_{0}=\min \left\{1,\left(\frac{1}{k_{2}}-\frac{1}{k_{1}}\right) \beta_{q}\left(k_{2} f\right)\left(1+\left(\rho^{*}\left(k_{2} f\right)\right)^{q}\right)^{\frac{1}{q}-1}\right\}>0$ and

$$
\frac{1}{k_{2}} s_{q}\left(\rho^{*}\left(k_{2} f\right)\right) \leq \frac{1}{k_{1}} s_{q}\left(\rho^{*}\left(k_{1} f\right)\right)-\varepsilon_{0}<\frac{1}{k_{1}} s_{q}\left(\rho^{*}\left(k_{1} f\right)\right),
$$

i.e., the function $k \rightarrow \frac{1}{k} s_{q}\left(\rho^{*}(k f)\right)$ is decreasing on $\left(0, k_{q}^{*}(f)\right)$.

(v) If $0<k_{1}<k_{2}<k_{q}^{* *}(f)$, let $f_{n}=v_{n}+\varphi, v_{n}$ be as in (5). Then

$I_{\Phi}\left(q_{+}\left(k_{2}\left|v_{n}\right|\right)\right) \cdot\left(I_{\Psi}\left(k_{2} v_{n}\right)+k_{2}\|\varphi\|\right)^{q-1} \leq 1$. Repeating the arguments used in the proof of condition (iv) with slight changes: $\beta_{q}\left(k_{2} f_{n}\right) \leq 0$ and $\varepsilon_{n}=0$ we get, passing with $n$ to infinity, that $\frac{1}{k_{2}} s_{q}\left(\rho^{*}\left(k_{2} f\right)\right) \leq \frac{1}{k_{1}} s_{q}\left(\rho^{*}\left(k_{1} f\right)\right)$.

(vi) Let $k_{q}^{* *}(f)<k_{1}<k_{2}<\left(\theta^{*}\right)^{-1}(f)$ and let $f_{n}=v_{n}+\varphi, v_{n}$ be as in (5). Then by Lemma 3.4, $I_{\Psi}\left(k_{i} v_{n}\right)<\infty$ and $I_{\Phi}\left(q_{+}\left(k_{i}\left|v_{n}\right|\right)\right)<\infty$ for $i=1,2$. Since $k_{q}^{* *}(f)<k_{1}$,

$$
0<\beta_{q}\left(k_{1} f\right)=I_{\Phi}\left(q_{+}\left(k_{1}|v|\right)\right)\left(I_{\Psi}\left(k_{1} v\right)+k_{1}\|\varphi\|\right)^{q-1}-1<\infty .
$$

Thus, for every $n \in N$ sufficiently large,

$$
0<\beta_{q}\left(k_{1} f_{n}\right)=I_{\Phi}\left(q_{+}\left(k_{1}\left|v_{n}\right|\right)\right)\left(I_{\Psi}\left(k_{1} v_{n}\right)+k_{1}\|\varphi\|\right)^{q-1}-1<\infty .
$$

Let $\varepsilon_{n}=\min \left\{1,\left(\frac{1}{k_{1}}-\frac{1}{k_{2}}\right) \beta_{q}\left(k_{1} f_{n}\right)\left(1+\left(\rho^{*}\left(k_{1} f_{n}\right)\right)^{q}\right)^{\frac{1}{q}-1}\right\}$. In an analogous way as above, for every sufficiently large $n \in N$, we get 


$$
\begin{aligned}
\frac{1}{k_{1}} s_{q}\left(\rho^{*}\left(k_{1} f_{n}\right)\right) & \\
= & \frac{\left(\rho^{*}\left(k_{1} f_{n}\right)\right)^{q-1}\left(\int_{G}\left|v_{n}(t)\right| q_{+}\left(k_{1}\left|v_{n}(t)\right|\right) \mathrm{d} t+\|\varphi\|\right)-\frac{1}{k_{1}} \beta_{q}\left(k_{1} f_{n}\right)}{\left(1+\left(\rho^{*}\left(k_{1} f_{n}\right)\right)^{q}\right)^{1-\frac{1}{q}}} \\
\leq & \frac{\left(\rho^{*}\left(k_{1} f_{n}\right)\right)^{q-1}\left(\int_{G}\left|v_{n}(t)\right| q_{+}\left(k_{1}\left|v_{n}(t)\right|\right) \mathrm{d} t+\|\varphi\|\right)-\frac{1}{k_{2}} \beta_{q}\left(k_{1} f_{n}\right)}{\left(1+\left(\rho^{*}\left(k_{1} f_{n}\right)\right)^{q}\right)^{1-\frac{1}{q}}}-\varepsilon_{n} \\
= & \frac{1+\left(\rho^{*}\left(k_{1} f_{n}\right)\right)^{q-1}\left(\int_{G} k_{2}\left|v_{n}(t)\right| q_{+}\left(k_{1}\left|v_{n}(t)\right|\right) \mathrm{d} t+k_{2}\|\varphi\|-I_{\Phi}\left(q_{+}\left(k_{1}\left|v_{n}\right|\right)\right)\right.}{k_{2}\left(1+\left(I_{\Psi}\left(k_{1} v_{n}\right)+k_{1}\|\varphi\|\right)^{q}\right)^{1-\frac{1}{q}}} \\
\leq & \frac{1+\left(\rho^{*}\left(k_{1} f_{n}\right)\right)^{q-1}\left(I_{\Psi}\left(k_{2} v_{n}\right)+k_{2}\|\varphi\|\right)}{k_{2}\left(1+\left(I_{\Psi}\left(k_{1} v_{n}\right)+k_{1}\|\varphi\|\right)^{q}\right)^{1-\frac{1}{q}}}-\varepsilon_{n} \\
\leq & \frac{1}{k_{2}}\left(1+\left(\rho^{*}\left(k_{2} f_{n}\right)\right)^{q}\right)^{\frac{1}{q}}-\varepsilon_{n}=\frac{1}{k_{2}} s_{q}\left(\rho^{*}\left(k_{2} f_{n}\right)\right)-\varepsilon_{n} .
\end{aligned}
$$

Letting $n \rightarrow \infty$ we get $\varepsilon_{n} \rightarrow \varepsilon_{0}=\min \left\{1,\left(\frac{1}{k_{1}}-\frac{1}{k_{2}}\right) \beta_{q}\left(k_{1} f\right)\left(1+\rho^{*}\left(k_{1} f\right)^{q}\right)^{\frac{1}{q}-1}\right\}>0$, so

$$
\frac{1}{k_{1}} s_{q}\left(\rho^{*}\left(k_{1} f\right)\right) \leq \frac{1}{k_{2}} s_{q}\left(\rho^{*}\left(k_{2} f\right)\right)-\varepsilon_{0}<\frac{1}{k_{2}} s_{q}\left(\rho^{*}\left(k_{2} f\right)\right),
$$

i.e., the function $k \rightarrow \frac{1}{k} s_{q}\left(\rho^{*}(k f)\right)$ is increasing on $\left(k_{q}^{* *}(f),\left(\theta^{*}\right)^{-1}(f)\right)$.

(vii) Let $k_{q}^{*}(f)<k_{1}<k_{2}<\left(\theta^{*}\right)^{-1}(f)$ and let $f_{n}=v_{n}+\varphi, v_{n}$ be as in (5). Then $\beta_{q}\left(k_{2} f\right) \stackrel{q}{=} I_{\Phi}\left(q_{+}\left(k\left|v_{n}\right|\right)\right) \cdot\left(I_{\Psi}\left(k v_{n}\right)+k\|\varphi\|\right)^{q-1}-1 \geq 0$. Repeating the arguments used in the proof of condition (vi) with slight changes: $\varepsilon_{n}=0$ we get, passing with $n$ to infinity, that $\frac{1}{k_{1}} s_{q}\left(\rho^{*}\left(k_{1} f\right)\right) \leq \frac{1}{k_{2}} s_{q}\left(\rho^{*}\left(k_{2} f\right)\right)$.

Theorem 3.6 All conditions of Theorem 3.5 hold true for $q=\infty$ and every $f \in\left(L_{\Phi, 1}\right)^{*} \backslash\{0\}$.

Proof We need to prove conditions (iv)-(vii) only.

Hence

(iv) Let $0<k_{1}<k_{2}<k_{\infty}^{*}(f)$. Then $\rho^{*}\left(k_{1} f\right) \leq \rho^{*}\left(k_{2} f\right) \leq 1$, because $\beta_{\infty}\left(k_{2} f\right)<0$.

$$
\begin{aligned}
\frac{1}{k_{2}} s_{\infty}\left(\rho^{*}\left(k_{2} f\right)\right) & =\frac{1}{k_{2}} \max \left\{1, \rho^{*}\left(k_{2} f\right)\right\} \\
& <\frac{1}{k_{1}} \max \left\{1, \rho^{*}\left(k_{1} f\right)\right\} \\
& =\frac{1}{k_{1}} s_{\infty}\left(\rho^{*}\left(k_{1} f\right)\right) .
\end{aligned}
$$

(v) Let $0<k_{1}<k_{2}<k_{\infty}^{* *}(f)$ and let $f_{n}=v_{n}+\varphi, v_{n}$ be as in (5). If $\rho^{*}\left(k_{2} f_{n}\right) \leq 1$ then $\frac{1}{k_{2}} s_{\infty}\left(\rho^{*}\left(k_{2} f\right)\right)<\frac{1}{k_{1}} s_{\infty}\left(\rho^{*}\left(k_{1} f\right)\right)$ by (iv). 
Assume $\rho^{*}\left(k_{2} f_{n}\right)>1$. Then, since $\beta_{\infty}\left(k_{2} f_{n}\right) \leq \beta_{\infty}\left(k_{2} f\right) \leq 0$, we get $I_{\Phi}\left(q_{+}\left(k_{2}\left|v_{n}\right|\right)\right)=0$. Thus, applying the Young Inequality, we obtain

$$
\begin{aligned}
\frac{1}{k_{2}} \rho^{*}\left(k_{2} f_{n}\right) & =\frac{1}{k_{2}}\left(\int_{G} k_{2}\left|v_{n}(t)\right| q_{+}\left(k_{2}\left|v_{n}(t)\right|\right) \mathrm{d} t-I_{\Phi}\left(q_{+}\left(k_{2}\left|v_{n}\right|\right)\right)+k_{2}\|\varphi\|\right) \\
& =\int_{G}\left|v_{n}(t)\right| q_{+}\left(k_{2}\left|v_{n}(t)\right|\right) \mathrm{d} t-\frac{1}{k_{2}} I_{\Phi}\left(q_{+}\left(k_{2}\left|v_{n}\right|\right)\right)+\|\varphi\| \\
& =\int_{G}\left|v_{n}(t)\right| q_{+}\left(k_{2}\left|v_{n}(t)\right|\right) \mathrm{d} t+\|\varphi\| \\
& =\int_{G}\left|v_{n}(t)\right| q_{+}\left(k_{2}\left|v_{n}(t)\right|\right) \mathrm{d} t-\frac{1}{k_{1}} I_{\Phi}\left(q_{+}\left(k_{2}\left|v_{n}\right|\right)\right)+\|\varphi\| \\
& \leq \frac{1}{k_{1}} I_{\Psi}\left(k_{1} v_{n}\right)+\|\varphi\|=\frac{1}{k_{1}} \rho^{*}\left(k_{1} f_{n}\right) .
\end{aligned}
$$

Hence, we have

$$
\begin{aligned}
\frac{1}{k_{2}} s_{\infty}\left(\rho^{*}\left(k_{2} f_{n}\right)\right) & =\frac{1}{k_{2}} \max \left\{1, \rho^{*}\left(k_{2} f_{n}\right)\right\} \\
& \leq \frac{1}{k_{1}} \max \left\{1, \rho^{*}\left(k_{1} f_{n}\right)\right\}=\frac{1}{k_{1}} s_{\infty}\left(\rho^{*}\left(k_{1} f_{n}\right)\right) .
\end{aligned}
$$

Letting $n \rightarrow \infty$, we obtain $\frac{1}{k_{2}} s_{\infty}\left(\rho^{*}\left(k_{2} f\right)\right) \leq \frac{1}{k_{1}} s_{\infty}\left(\rho^{*}\left(k_{1} f\right)\right)$.

(vi) Let $k_{\infty}^{* *}(f)<k_{1}<k_{2}<\left(\theta^{*}\right)^{-1}(f)$ and let $f_{n}=v_{n}+\varphi, v_{n}$ be as in (5). Since $\beta_{\infty}\left(k_{1} f\right)>0$, we have $\rho^{*}\left(k_{1} f\right)>1$ and $I_{\Phi}\left(q_{+}\left(k_{1}|v|\right)\right)>0$. Let $\varepsilon_{n}=\min \left\{1,\left(\frac{1}{k_{1}}-\frac{1}{k_{2}}\right) I_{\Phi}\left(q_{+}\left(k_{1}\left|v_{n}\right|\right)\right)\right\}$. Then, by the Young Inequality,

$$
\begin{aligned}
\frac{1}{k_{1}} \rho^{*}\left(k_{1} f_{n}\right) & =\frac{1}{k_{1}}\left(\int_{G} k_{1}\left|v_{n}(t)\right| q_{+}\left(k_{1}\left|v_{n}(t)\right|\right) \mathrm{d} t-I_{\Phi}\left(q_{+}\left(k_{1}\left|v_{n}\right|\right)\right)+k_{1}\|\varphi\|\right) \\
& =\int_{G}\left|v_{n}(t)\right| q_{+}\left(k_{1}\left|v_{n}(t)\right|\right) \mathrm{d} t-\frac{1}{k_{1}} I_{\Phi}\left(q_{+}\left(k_{1}\left|v_{n}\right|\right)\right)+\|\varphi\| \\
& =\int_{G}\left|v_{n}(t)\right| q_{+}\left(k_{1}\left|v_{n}(t)\right|\right) \mathrm{d} t-\frac{1}{k_{2}} I_{\Phi}\left(q_{+}\left(k_{1}\left|v_{n}\right|\right)\right)-\varepsilon_{n}+\|\varphi\| \\
& \leq \frac{1}{k_{2}}\left(I_{\Psi}\left(k_{2} v_{n}\right)\right)-\varepsilon_{n}+\|\varphi\|=\frac{1}{k_{2}} \rho^{*}\left(k_{2} f_{n}\right)-\varepsilon_{n} .
\end{aligned}
$$

Passing with $n$ to infinity, we get $\varepsilon_{n} \rightarrow \varepsilon_{0}=\left(\frac{1}{k_{1}}-\frac{1}{k_{2}}\right) I_{\Phi}\left(q_{+}\left(k_{1}|v|\right)\right)>0$, so $\frac{1}{k_{1}} s_{\infty}\left(\rho^{*}\left(k_{1} f\right)\right) \leq \frac{1}{k_{2}} s_{\infty}\left(\rho^{*}\left(k_{2} f\right)\right)-\varepsilon_{0}<\frac{1}{k_{2}} s_{\infty}\left(\rho^{*}\left(k_{2} f\right)\right)$.

(vii) Let $k_{\infty}^{*}(f)<k_{1}<k_{2}<\left(\theta^{*}\right)^{-1}(f)$ and let $f_{n}=v_{n}+\varphi, v_{n}$ be as in (5). Since $\beta_{\infty}\left(k_{1} f\right) \geq 0$, we have $\rho^{*}\left(k_{1} f\right)>1$ and $I_{\Phi}\left(q_{+}\left(k_{1}|v|\right)\right) \geq 0$. Repeating the arguments used in the proof of condition (vi) with $\varepsilon_{n}=0$. We get, passing with $n$ to infinity, that $\frac{1}{k_{1}} s_{\infty}\left(\rho^{*}\left(k_{1} f\right)\right) \leq \frac{1}{k_{2}} s_{\infty}\left(\rho^{*}\left(k_{2} f\right)\right)$.

As an immediate consequence of Theorems 3.5 and 3.6 we get the following theorem. 
Theorem 3.7 For every $1 \leq q \leq \infty$ and each $f \in\left(L_{\Phi, p}\right)^{*} \backslash\{0\}$ the following conditions hold.

(i) If $k_{q}^{*}(f)=k_{q}^{* *}(f)=\infty$, then $\|f\|_{\Psi, q}^{*}=\lim _{k \rightarrow \infty} \frac{1}{k} s_{q}\left(\rho^{*}(k f)\right)$.

(ii) If $k_{q}^{*}(f)<k_{q}^{* *}(f)=\infty$, then $\|f\|_{\Psi, q}^{*}$ is attained at every $k \in\left[k_{q}^{*}(f), \infty\right)$.

(iii) If $k_{q}^{* *}(f)<\infty$, then $\|f\|_{\Psi, q}^{*}$ is attained at every $k \in\left[k_{q}^{*}(f), k_{q}^{* *}(f)\right]$.

Theorem 3.8 Every Orlicz function $\Psi$ with $b_{\Psi}<\infty$ is $k_{q}^{*}$-finite, i.e., $K_{q}(f) \neq \emptyset(1 \leq q \leq \infty)$.

Proof If $b_{\Psi}<\infty$, then $\left(\theta^{*}\right)^{-1}(f)<\infty$ for every $f \in\left(L_{\Phi, p}\right)^{*} \backslash\{0\}$, evidently,

$$
\|f\|_{\Psi, q}^{*}=\frac{1}{k_{q}^{* *}(f)} s_{q}\left(\rho^{*}\left(k_{q}^{* *}(f) f\right)\right)=\inf _{k>0} \frac{1}{k} s_{q}\left(\rho^{*}(k f)\right)<\infty .
$$

Hence, $k_{q}^{*}(f) \leq k_{q}^{* *}(f) \leq\left(\theta^{*}\right)^{-1}(f)$. Thus, every Orlicz function is $K_{q}(f) \neq \emptyset$ as long as $b_{\Psi}<\infty$.

Theorem 3.9 For all $f \in\left(L_{\Phi, p}\right)^{*} \backslash\{0\}(1 \leq p \leq \infty)$ is of the form (2).

(i) $q=1$ i.e., $p=\infty$. If $I_{\Phi}\left(b_{\Phi} \chi_{\operatorname{supp}(v)}\right) \geq 1$, then $K_{1}(f) \neq \emptyset$.

(ii) $1<q<\infty, \frac{1}{p}+\frac{1}{q}=1$. If $\varphi \neq 0$, then for every Orlicz function $\Psi, K_{q}(f) \neq \emptyset$. If $\varphi=0$ and $\Psi$ is not linear on $[0, \infty)$, then $K_{q}(f) \neq \emptyset$.

(iii) $q=\infty$ i.e., $p=1$. For every Orlicz function $\Phi, K_{\infty}(f) \neq \emptyset$.

Proof (i) When $q=1, \beta_{1}(f)=I_{\Phi}\left(q_{+}(|v|)\right)-1$. If $I_{\Phi}\left(b_{\Phi} \chi_{\operatorname{supp}(v)}\right) \geq 1$, there exists $k>0$ such that $\beta_{1}(k f) \geq 0$. By the definition of $k_{1}^{*}(f)$, we have $k_{1}^{*}(f)<\infty$, i.e., $K_{1}(f) \neq \emptyset$.

(ii) If $\varphi \neq 0$, then $\|\varphi\|>0$. We have $\rho^{*}(k f)=I_{\Psi}(k v)+k\|\varphi\| \rightarrow \infty$ as $k \rightarrow \infty$. Thus, there exists $k>0$, such that $I_{\Phi}\left(q_{+}(k|v|)\right)\left(\rho^{*}(k f)\right)^{q-1}>1$. Hence, $k_{q}^{*}(f)<\infty$ i.e., $K_{q}(f) \neq \emptyset$.

If $\varphi=0$. The proof is similar to Theorem 4.3 in [9], so we omit it here.

(iii) Since $\rho^{*}\left(\frac{f}{\|f\|_{\Psi, \infty}^{*}}\right) \leq 1$, we have $\beta_{\infty}\left(\frac{f}{\|f\|_{\Psi, \infty}^{*}}\right)=-1$, so $\frac{1}{\|f\|_{\Psi, \infty}^{*}} \leq k_{\infty}^{*}(f)$. Suppose $\frac{1}{\|f\|_{\Psi, \infty}^{*}}<k<k_{\infty}^{*}(f)$ for some $k>0$. Then $\beta_{\infty}(k f)<0$, so $\rho^{*}(k f) \leq 1$, whence $k<\frac{1}{\|f\|_{\Psi, \infty}^{*}}$ a contradiction. Thus $0<\frac{1}{\|f\|_{\Psi, \infty}^{*}}=k_{\infty}^{*}(f)$. That is $K_{\infty}(f) \neq \emptyset$.

\section{Bounded linear functionals}

Lemma 4.1 (Minkowski inequality) For any sequences $\left\{\xi_{k}\right\},\left\{\eta_{k}\right\} \subset R$, we have

(i) $\left(\sum_{k}\left|\xi_{k}+\eta_{k}\right|_{1}^{q}\right)^{\frac{1}{q}} \leq\left(\sum_{k}\left|\xi_{k}\right|^{q}\right)^{\frac{1}{q}}+\left(\sum_{k}\left|\eta_{k}\right|^{q}\right)^{\frac{1}{q}}$ for every $1 \leq q<\infty$,

(ii) $\left(1+(u+v)^{q}\right)^{q} \leq\left(1+u^{q}\right)^{\bar{q}}+v$ for all $u, v \geq 0$ and every $1 \leq q<\infty$, 
(iii) $\left(1+\left(\frac{u+v}{2}\right)^{q}\right)^{\frac{1}{q}} \leq \frac{1}{2}\left(1+u^{q}\right)^{\frac{1}{q}}+\frac{1}{2}\left(1+v^{q}\right)^{\frac{1}{q}}$ for all $u, v \geq 0$ and every $1 \leq q<\infty$

Proof The part (i) follows directly from the Minkowski Inequality. If we put $\xi_{1}=1$, $\eta_{1}=0, \xi_{2}=u, \eta_{2}=v$, then we get the condition (ii) for $1 \leq q<\infty$. Similarly, we put $\xi_{1}=\frac{1}{2}, \eta_{1}=\frac{1}{2}, \xi_{2}=\frac{u}{2}, \eta_{2}=\frac{v}{2}$, then we get the condition (iii) for $1 \leq q<\infty$.

Theorem 4.2 Let $\Phi, \Psi$ be the Orlicz functions complementary in the sense of Young that take finite values only. Assuming the p-Amemiya norm $\|\cdot\|_{\Phi, p}$ is $k_{p}^{*}$-finite. Let $f \in\left(L_{\Phi, p}\right)^{*}(1 \leq p \leq \infty)$, $f$ have the unique decomposition $f=v+\varphi$ where $v \in L_{\Psi, q}, \frac{1}{p}+\frac{1}{q}=1, \varphi \in F$. Then

$$
\|f\|=\|f\|_{\Psi, q}^{*}= \begin{cases}\inf _{k>0} \frac{1}{k} s_{q}\left(\rho^{*}(k f)\right), & \text { for } 1 \leq q<\infty, \\ \inf _{k>0} \frac{1}{k} \max \left\{1, \rho^{*}(k f)\right\}, & \text { for } q=\infty .\end{cases}
$$

Proof By the definition of $\|f\|_{\Psi, q}^{*}$, we have $\|f\|_{\Psi, 1}^{*}=\|f\|_{\Psi}^{\circ}=\|v\|_{\Psi}^{\circ}+\|\varphi\|_{\Psi}^{\circ}$ and $\|f\|_{\Psi, \infty}^{*}=\|f\|_{\Psi}=\inf _{l>0}\left\{\frac{1}{l}, I_{\Psi}(l v)+l\|\varphi\| \leq 1\right\}$. So, we will prove the cases of $1<q<\infty$.

For any $f \in\left(L_{\Phi, p}\right)^{*}$, if $\varphi=0$ then Orlicz space $L_{\Phi, p}$ is order continuous, i.e, $L_{\Phi, p}=E_{\Phi, p}$. By Lemma 2.7, We have $\left(E_{\Phi, p}\right)^{*}=L_{\Psi, q}$. The case has been discussed. So we assume $\varphi \neq 0$. By Theorem 3.9, we know $K_{q}(f) \neq \emptyset$.

$\forall l>0, \forall u \in S\left(L_{\Phi, p}\right)$, take $k \in K_{p}(u)$, by the Young Inequality and the definition of conjugate outer functions, we have

$$
\begin{aligned}
l f(u) & =\frac{1}{k}\left(\langle k u, l v>+l \varphi(k u))=\frac{1}{k}\left(\int_{G} k u(t) l v(t) \mathrm{d} t+l \varphi(k u)\right)\right. \\
& \leq \frac{1}{k}\left(I_{\Phi}(k u)+I_{\Psi}(l v)+l\|\varphi\|\right) \leq \frac{1}{k} s_{p}\left(I_{\Phi}(k u)\right) \cdot s_{q}\left(\rho^{*}(l f)\right)=s_{q}\left(\rho^{*}(l f)\right),
\end{aligned}
$$

where $\varphi(k u) \leq\|\varphi\|$ by Lemma $2.9 \quad$ and $\quad I_{\Phi}(k u)=\left(k^{p}-1\right)^{\frac{1}{p}}<\infty$. So $f(u) \leq \frac{1}{l} s_{q}\left(\rho^{*}(l f)\right)$. Since $u$ and $l$ are arbitrary, we deduce that

$$
\|f\| \leq \inf _{l>0} \frac{1}{l} s_{q}\left(\rho^{*}(l f)\right)=\|f\|_{\Psi, q}^{*} .
$$

Take $l \in K_{q}(f)$, for any $\varepsilon>0$, take $k_{0} \in K_{p}\left(q_{+}(l v)\right)$ and choose $y \in S\left(L_{\Phi, p}\right)$ such that $\|\varphi\|-\varepsilon<\varphi\left(\frac{y}{k_{0}}\right)$. Select $\delta>0$ such that

$$
\mu(E)<\delta \Rightarrow \int_{E} l\left|q_{+}(l v(t)) \cdot v(t)\right| \mathrm{d} t<\varepsilon,
$$

then pick $k>0$ such that $\mu H<\delta$ and that

$$
\int_{H} \frac{l}{k_{0}}|y(t) v(t)| \mathrm{d} t<\varepsilon, \quad \int_{H} \Phi(y(t)) \mathrm{d} t<\varepsilon,
$$

where $H=\{t \in G:|y(t)|>k\}$. Define 


$$
u(t)= \begin{cases}q_{+}(l v(t)), & t \in G \backslash H, \\ \frac{y(t)}{k_{0}}, & t \in H .\end{cases}
$$

Then by Lemma 4.1(ii), we have

$$
\begin{aligned}
\|u\|_{\Phi, p} & =\inf _{k>0} \frac{1}{k}\left(1+I_{\Phi}^{p}(k u)\right)^{\frac{1}{p}} \\
& =\inf _{k>0} \frac{1}{k}\left(1+\left(\int_{G \backslash H} \Phi\left(k q_{+}(l v(t)) \mathrm{d} t+\int_{H} \Phi\left(k y(t) / k_{0}\right) \mathrm{d} t\right)^{p}\right)^{\frac{1}{p}}\right. \\
& \leq \frac{1}{k_{0}}\left(1+\left(\int_{G \backslash H} \Phi\left(k_{0} q_{+}(l v(t)) \mathrm{d} t+\int_{H} \Phi\left(k_{0} y(t) / k_{0}\right) \mathrm{d} t\right)^{p}\right)^{\frac{1}{p}}\right. \\
& \leq \frac{1}{k_{0}}\left(1+\left(I_{\Phi}\left(k_{0} q_{+}(l v)\right)+\varepsilon\right)^{p}\right)^{\frac{1}{p}} \\
& \leq \frac{1}{k_{0}}\left(1+I_{\Phi}^{p}\left(k_{0} q_{+}(l v)\right)\right)^{\frac{1}{p}}+\frac{\varepsilon}{k_{0}}=\left\|q_{+}(l v)\right\|_{\Phi, p}+\frac{\varepsilon}{k_{0}} .
\end{aligned}
$$

For the arbitrary of $\varepsilon$, we obtain $\|u\|_{\Phi, p} \leq\left\|q_{+}(l v)\right\|_{\Phi, p}$.

Since $l \in K_{q}(f)$, that means $I_{\Phi}\left(q_{+}(l v)\right) \cdot\left(\rho^{*}(l f)\right)^{q-1}=1$. By the Young Inequality and Lemma 2.6, we have

$$
\begin{aligned}
\|f\| & \geq \frac{1}{\|u\|_{\Phi, p}} f(u)=\frac{1}{\|u\|_{\Phi, p}}\left(f\left(q_{+}(l v) \chi_{G \backslash H}\right)+f\left(y k_{0}^{-1} \cdot \chi_{H}\right)\right) \\
& =\frac{<l v, q_{+}(l v) \chi_{G \backslash H}>+<l v, y k_{0}^{-1} \cdot \chi_{H}>+l \varphi\left(q_{+}(l v) \chi_{G \backslash H}\right)+l \varphi\left(y k_{0}^{-1}\right)}{l\|u\|_{\Phi, p}} \\
& \geq \frac{<l v, q_{+}(l v)>-<l v, q_{+}(l v) \chi_{H}>+<l v, y k_{0}^{-1} \cdot \chi_{H}>+l \varphi\left(y k_{0}^{-1}\right)}{l\|u\|_{\Phi, p}} \\
> & \frac{1}{l\|u\|_{\Phi, p}}\left(I_{\Phi}\left(q_{+}(l v)\right)+I_{\Psi}(l v)-2 \varepsilon+l(\|\varphi\|-\varepsilon)\right) \\
& =\frac{1}{l\|u\|_{\Phi, p}}\left(s_{p}\left(I_{\Phi}\left(q_{+}(l v)\right)\right) \cdot s_{q}\left(\rho^{*}(l f)\right)-(l+2) \varepsilon\right) \\
& =\frac{1}{\|u\|_{\Phi, p}}\left(s_{p}\left(I_{\Phi}\left(q_{+}(l v)\right)\right)\|f\|_{\Psi, q}^{*}-\left(1+2 l^{-1}\right) \varepsilon\right) \\
& \geq \frac{\left\|q_{+}(l v)\right\|_{\Phi, p}}{\|u\|_{\Phi, p}}\|f\|_{\Psi, q}^{*}-\frac{\left(1+2 l^{-1}\right) \varepsilon}{\|u\|_{\Phi, p}} \geq\|f\|_{\Psi, q}^{*}-\frac{\left(1+2 l^{-1}\right) \varepsilon}{\|u\|_{\Phi, p}} .
\end{aligned}
$$

Letting $\varepsilon \rightarrow 0$, we get $\|f\| \geq\|f\|_{\Psi, q}^{*}$, combine $\|f\| \leq\|f\|_{\Psi, q}^{*}$, we have $\|f\|=\|f\|_{\Psi, q}^{*}$.

Theorem 4.3 For any $\varphi \in F \backslash\{0\}$ is not norm attainable on $S\left(L_{\Phi, p}\right), 1 \leq p<\infty$.

Proof For any $u \in S\left(L_{\Phi, p}\right), 1 \leq p<\infty$ we have 


$$
\varphi(u) \leq\|\varphi\| \cdot\|u\|_{\Phi}<\|\varphi\| \cdot\|u\|_{\Phi, p}=\|\varphi\| .
$$

Theorem 4.4 Assuming the p-Amemiya norm $\|\cdot\|_{\Phi, p}$ is $k_{p}^{*}$-finite $(1 \leq p \leq \infty)$, $f \in\left(L_{\Phi, p}\right)^{*} \backslash\{0\}$ where $f=v+\varphi$ is norm attainable at $u \in S\left(L_{\Phi, p}\right)$ if and only if:

(a) $q=1, p=\infty$, for any $l \in K_{1}(f)$. Then

(i) $\|\varphi\|=\varphi(u)$,

(ii) $\int_{G} l v(t) u(t) \mathrm{d} t=I_{\Phi}(u)+I_{\Psi}(l v)$, and

(iii) $I_{\Phi}(u)=1$.

(b) $1<p, q<\infty, \frac{1}{p}+\frac{1}{q}=1$, for any $k \in K_{p}(u), l \in K_{q}(f)$. Then

(i) $\|\varphi\|=\varphi(k u)$,

(ii) $\int_{G} k u(t) l v(t) \mathrm{d} t=I_{\Phi}(k u)+I_{\Psi}(l v)$, and

(iii) $I_{\Phi}^{P-1}(k u) \rho^{*}(l f)=I_{\Phi}(k u)\left(\rho^{*}(l f)\right)^{q-1}=1$.

(c) $q=\infty, p=1$, for any $k \in K_{1}(u)$. Then

(i) $\|\varphi\|=\varphi(k u)$,

(ii) $\int_{G} k u(t) \frac{v(t)}{\|f\|_{\Psi_{,}}^{*}} \mathrm{~d} t=I_{\Phi}(k u)+I_{\Psi}\left(\frac{v}{\|f\|_{\Psi_{\infty}}^{*}}\right)$, and

(iii) $\rho^{*}\left(\frac{f}{\|f\|_{\Psi, \infty}^{*}}\right) \stackrel{\Psi, \infty}{=} 1$.

Proof When $q=1$ or $q=\infty$, we have $\|f\|_{\Psi, 1}^{*}=\|f\|_{\Psi}^{\circ}$ and $\|f\|_{\Psi, \infty}^{*}=\|f\|_{\Psi}$. The conclusions of (a) and (c) are known (see [6, Theorem 1.76, 1.77]). We need to prove case (b) only.

For any $k \in K_{p}(u), l \in K_{q}(f)$,

$$
\begin{aligned}
f(u) & =\frac{1}{l k}(<l v, k u>+l \varphi(k u)) \leq \frac{1}{l k}\left(I_{\Phi}(k u)+I_{\Psi}(l v)+l \varphi(k u)\right) \\
& \leq \frac{1}{l k}\left(I_{\Phi}(k u)+I_{\Psi}(l v)+l\|\varphi\|\right)=\frac{1}{l k}\left(I_{\Phi}(k u)+\rho^{*}(l f)\right) \\
& \leq \frac{1}{k} s_{p}\left(I_{\Phi}(k u)\right) \cdot \frac{1}{l} s_{q}\left(\rho^{*}(l f)\right)=\|u\|_{\Phi, p} \cdot\|f\|_{\Psi, q}^{*}=\|f\|_{\Psi, q}^{*},
\end{aligned}
$$

where $\varphi(k u) \leq\|\varphi\|$ holds by Lemma 2.9 and $I_{\Phi}(k u)=\left(k^{p}-1\right)^{\frac{1}{p}}<\infty$. Suppose that (i), (ii) and (iii) are satisfied, then all inequalities become equalities. Hence, $f$ is norm attainable at $u \in S\left(L_{\Phi, p}\right)$.

Conversely, let $f=v+\varphi \in\left(L_{\Phi, p}\right)^{*}$ be norm attainable at $u \in S\left(L_{\Phi, p}\right)$. We have 


$$
\begin{aligned}
0 & =f(u)-\|f\|_{\Psi, q}^{*} \cdot\|u\|_{\Phi, p} \\
& =\frac{1}{k l}(<l v, k u>+l \varphi(k u))-\frac{1}{l} s_{q}\left(\rho^{*}(l f)\right) \cdot \frac{1}{k} s_{p}\left(I_{\Phi}(k u)\right) . \\
& \leq \frac{1}{l k}\left(I_{\Phi}(k u)+I_{\Psi}(l v)+l \varphi(k u)\right)-\frac{1}{l} s_{q}\left(\rho^{*}(l f)\right) \cdot \frac{1}{k} s_{p}\left(I_{\Phi}(k u)\right) \\
& \leq \frac{1}{l k}\left(I_{\Phi}(k u)+I_{\Psi}(l v)+l \varphi(k u)\right)-\frac{1}{l k}\left(I_{\Phi}(k u)+I_{\Psi}(l v)+l\|\varphi\|\right) \\
& =\frac{1}{l k}(l \varphi(k u)-l\|\varphi\|) \leq 0 .
\end{aligned}
$$

Then we obtain the condition (i). By the Young Inequality, the condition (ii) holds. By Lemma 2.6, we have the condition (iii).

Theorem 4.5 Assuming the $p$-Amemiya norm is $k_{p}^{*}$-finite. Let $u \in L_{\Phi, p}(1 \leq p \leq \infty)$. Then $v \in S\left(L_{\Psi, q}\right)$ is a supporting functional of $u$ if and only if:

(a) $q=1, p=\infty$. Then

(i) $\quad I_{\Phi}\left(\frac{u}{\|u\|_{\Phi, \infty}}\right)=1$, and

(ii) $\quad v=\frac{w}{\|w\|_{\Psi, 1}} \cdot \operatorname{sign} u$, for some $w$ satisfying $p_{-}\left(\frac{u(t)}{\|u\|_{\Phi, \infty}}\right) \leq w(t) \leq p_{+}\left(\frac{u(t)}{\|u\|_{\Phi, \infty}}\right)$,

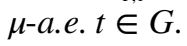

(b) $1<p, q<\infty, \frac{1}{p}+\frac{1}{q}=1$. Then

(i) $\quad v=\frac{w}{\|w\|_{\Psi, q}} \cdot \operatorname{sign} u$, for some $w$ satisfying $p_{-}(k u(t)) \leq w(t) \leq p_{+}(k u(t)), \mu$ -a.e. $t \in G, k \in K_{p}(u)$, and

(ii) $\quad I_{\Phi}(k u) \cdot I_{\Psi}^{q-1}(w)=1$.

(c) $q=\infty, p=1$. Then

(i) $\quad I_{\Psi}(v)=1$, and

(ii) $\quad p_{-}(k u(t)) \leq v(t) \leq p_{+}(k u(t))$, $\mu$-a.e. $t \in G, k \in K_{p}(u)$.

Proof It is well known $\|f\|_{\Psi, 1}^{*}=\|f\|_{\Psi}^{\circ}$ and $\|f\|_{\Psi, \infty}^{*}=\|f\|_{\Psi}$, and the conclusions of (a) and (c) are obtained (see [6, Theorem 1.78, 1.80]). We need to prove case (b) only.

Sufficiency. Suppose $\langle v, u\rangle=\|v\|_{\Psi, q} \cdot\|u\|_{\Phi, p}=\|u\|_{\Phi, p}$. Then $v(t) \cdot u(t) \geq 0, \mu$ -a.e. $t \in G$. Given $v_{0}$ is norm attainable at $u$, take $k \in K_{p}(u), l \in K_{q}\left(v_{0}\right)$, by Theorem 4.4(b-ii) and the Young Inequality, we have

$$
p_{-}(k u(t)) \leq l\left|v_{0}(t)\right| \leq p_{+}(k u(t)), \mu \text {-a.e. } t \in G .
$$

By Theorem 4.4(b-iii) and $\varphi=0$, we have $I_{\Phi}(k u) \cdot I_{\Psi}^{q-1}\left(l v_{0}\right)=1$. Hence, $w=l\left|v_{0}\right|$ is as required. Now let 


$$
v=\frac{w}{\|w\|_{\Psi, q}} \cdot \operatorname{sign} u, \quad p_{-}(k u(t)) \leq w(t) \leq p_{+}(k u(t)) .
$$

Then by the Young Inequality and the definition of $p$-Amemiya norm,

$$
\begin{aligned}
1 & \geq\left\langle v, \frac{u}{\|u\|_{\Phi, p}}\right\rangle=\frac{1}{\|w\|_{\Psi, q} \cdot\|u\|_{\Phi, p}}\langle w, u\rangle \\
& =\frac{1}{k\|w\|_{\Psi, q} \cdot\|u\|_{\Phi, p}}\left(I_{\Phi}(k u)+I_{\Psi}(w)\right) \\
& =\frac{s_{q}\left(I_{\Psi}(w)\right)}{\|w\|_{\Psi, q}} \cdot \frac{1}{\|u\|_{\Phi, p}} \frac{1}{k} s_{p}\left(I_{\Phi}(k u)\right) \geq \frac{\|w\|_{\Psi, q}}{\|w\|_{\Psi, q}}=1 .
\end{aligned}
$$

Necessity. If condition (i) fails, i.e., $l v_{0}(t)=w(t) \notin\left[p_{-}(k u(t)), p_{+}(k u(t))\right]$, by Theorem 4.4(b-ii) $v_{0}$ is not norm attainable at $u$. Hence, $v=\frac{w}{\|w\|_{\Psi, q}} \cdot \operatorname{sign} u$ is not a supporting functional of $u$.

If condition (ii) fails, i.e., $I_{\Phi}(k u) \cdot I_{\Psi}^{q-1}(w) \neq 1$. In an analogous way as above, by Theorem 4.4(b-iii) and $\varphi=0$, we have $v_{0}$ is not norm attainable at $u$. Hence, $v=\frac{w}{\|w\|_{\Psi, q}} \cdot \operatorname{sign} u$ is not a supporting functional of $u$.

\section{Smoothness}

Let $X$ be a Banach space. $u \in X$ is called a smooth point if it has a unique supporting functional $f_{u}$. If every $u \neq 0$ is a smooth point, then $X$ is called a smooth space. Criteria for smooth points of Orlicz function (sequence) spaces equipped with the Orlicz norm and Luxemburg norm were given in [5, 7, 13, 28]. Criteria for smoothness of Orlicz function (sequence) spaces equipped with the Orlicz norm and Luxemburg norm were given in $[4,16,26,27]$. In this section, we provide a characterization of smooth points in $L_{\Phi, p}(1 \leq p \leq \infty)$ and as a result, we give necessary and sufficient conditions for the smoothness of $L_{\Phi, p}$.

For any $u \in L_{\Phi, p}(1 \leq p \leq \infty)$, for each $n \in N$, set

$$
G(n)=\{t \in G:|u(t)| \leq n\}, \quad u_{n}(t)=u(t) \cdot \chi_{G_{n}(t)} .
$$

Lemma 5.1 [6] For any $u \in L_{\Phi}$,

$$
\lim _{n \rightarrow \infty}\left\|u-u_{n}\right\|_{\Phi}=\lim _{n \rightarrow \infty}\left\|u-u_{n}\right\|_{\Phi}^{o}=\theta(u),
$$

where $u_{n}$ is defined as in (6) and $\theta(u)=\inf \left\{\lambda>0, I_{\Phi}\left(\frac{u}{\lambda}\right)<\infty\right\}$.

By Lemma 5.1 and (1), we have

$$
\theta(u)=\lim _{n \rightarrow \infty}\left\|u-u_{n}\right\|_{\Phi, p}=\inf \left\{\lambda>0, I_{\Phi}\left(\frac{u}{\lambda}\right)<\infty\right\} .
$$


Lemma $5.2[6]$ Let $u \in L_{\Phi}$ and $\theta(u) \neq 0$. Then there exist two different singular functionals $\varphi_{i} \in S\left(L_{\Phi}\right)^{*}$ such that $\varphi_{i}(u)=\theta(u), i=1,2$.

Theorem 5.3 Let $u \in L_{\Phi, p}(1 \leq p \leq \infty)$ and for any singular functional $\varphi$. Then we have $\theta(u)<\left(k^{* *}(u)\right)^{-1}$ and $\varphi(u) \leq \theta(u) \cdot\|\varphi\|$.

Proof Let $u_{n}$ be defined as in (6), Then $u_{n} \in E_{\Phi, p}$. We have

$$
\left\|u-u_{n}\right\|_{\Phi, p}=\inf _{k>0} \frac{1}{k} s_{p}\left(I_{\Phi}\left(k\left(u-u_{n}\right)\right) \leq \frac{1}{k^{* *}(u)} s_{p}\left(I_{\Phi}\left(k^{* *}(u)\left(u-u_{n}\right)\right)<\infty .\right.\right.
$$

Letting $n \rightarrow \infty$, we have $\theta(u) \leq \frac{1}{k^{* *}(u)}$. Since $\varphi\left(E_{\Phi, p}\right)=0$ then

$$
\varphi(u)=\varphi\left(u-u_{n}\right) \leq\|\varphi\| \cdot\left\|u-u_{n}\right\|_{\Phi, p} .
$$

Letting $n \rightarrow \infty, \varphi(u) \leq\|\varphi\| \cdot \theta(u)$.

Theorem $5.4 u \in S\left(L_{\Phi, p}\right)(1 \leq p \leq \infty), u \neq 0$ and $\theta(u)<\frac{1}{k}, k \in K_{p}(u)$. Then the supporting functional of $u$ must be in $L_{\Psi, q}$ where $\frac{1}{p}+\frac{1}{q}=1$ and $\Psi$ is the function complementary to the Orlicz function $\Phi$ in the sense of Young.

Proof If $p=1$ or $p=\infty$, then [31] has given the proofs. We need to prove the cases of $1<p<\infty$.

Let $f$ be the supporting functional of $u$. Then $f$ has the unique decomposition $f=v+\varphi$ where $v \in L_{\Psi, q}(1<q<\infty), \varphi \in F$. Assuming $\varphi \neq 0$, then

$$
f(u)=\int_{G} u(t) v(t) \mathrm{d} t+\varphi(u) .
$$

For any $k \in K_{p}(u), l \in K_{q}(f)$, by Theorem 5.3, the Young Inequality and the definition of conjugate outer functions, we get

$$
\begin{aligned}
k l & =k l \int_{G} u(t) v(t) \mathrm{d} t+k l \varphi(u) \\
& \leq I_{\Phi}(k u)+I_{\Psi}(l v)+k l\|\varphi\| \theta(u) \\
& <I_{\Phi}(k u)+I_{\Psi}(l v)+l\|\varphi\| \\
& \leq s_{p}\left(I_{\Phi}(k u)\right) s_{q}\left(I_{\Psi}(l v)+l\|\varphi\|\right) \\
& =s_{p}\left(I_{\Phi}(k u)\right) s_{q}\left(\rho^{*}(l f)\right)=k l
\end{aligned}
$$

a contradiction.

Theorem 5.5 If $p_{-}(u)$ is continuous and $u \in L_{\Phi, p} \backslash\{0\}(1 \leq p \leq \infty)$ is a smooth point if and only if the supporting functional of $u$ must be in $L_{\Psi, q}$ where $\frac{1}{p}+\frac{1}{q}=1$ and $\Psi$ is the function complementary to the Orlicz function $\Phi$ in the sense of Young. 
Proof If $p=1$ or $p=\infty$, the conclusions have been proved in [26], so we omit them here. We need to prove the cases of $1<p<\infty$ only.

Sufficiency. Let $v_{0} \in S\left(L_{\Psi, q}\right)(1<q<\infty)$ be a supporting functional of $u$. If there is another supporting functional $f$ of $u$, and $f=v+\varphi, \varphi \neq 0$. Then $\frac{f+v_{0}}{2}=\frac{v+v_{0}}{2}+\frac{\varphi}{2}$ will be a supporting functional of $u$, too. Since

$$
\begin{aligned}
\left\|v_{0}\right\|_{\Psi, q} & =\inf _{k>0} \frac{1}{k}\left(1+I_{\Psi}^{q}\left(k v_{0}\right)\right)^{\frac{1}{q}}=1 \quad \text { and } \\
\|f\|_{\Psi, q}^{*} & =\inf _{k>0} \frac{1}{k}\left(1+\left(I_{\Psi}(k v)+k\|\varphi\|\right)^{q}\right)^{\frac{1}{q}}=1 .
\end{aligned}
$$

By the convexity of $\Psi$ and Lemma 4.1(iii), we have

$$
\begin{aligned}
1 & =\left\|\frac{f+v_{0}}{2}\right\|_{\Psi, q}^{*} \\
& =\inf _{k>0} \frac{1}{k}\left(1+\left(I_{\Psi}\left(k \frac{v+v_{0}}{2}\right)+k\left\|\frac{\varphi}{2}\right\|\right)^{q}\right)^{\frac{1}{q}} \\
& \leq \inf _{k>0} \frac{1}{k}\left(1+\left(\left(\frac{I_{\Psi}(k v)}{2}\right)+\left(\frac{I_{\Psi}\left(k v_{0}\right)}{2}\right)+\frac{k}{2}\|\varphi\|\right)^{q}\right)^{\frac{1}{q}} \\
& \leq \inf _{k>0} \frac{1}{k}\left(\frac{1}{2}\left(1+I_{\Psi}^{q}\left(k v_{0}\right)\right)^{\frac{1}{q}}+\frac{1}{2}\left(1+\left(I_{\Psi}(k v)+k\|\varphi\|\right)^{q}\right)^{\frac{1}{q}}\right) \\
& =\frac{\left\|v_{0}\right\|_{\Psi, q}}{2}+\frac{\|f\|_{\Psi, q}^{*}}{2}=1 .
\end{aligned}
$$

Hence, $I_{\Psi}\left(k \frac{v+v_{0}}{2}\right)=\frac{I_{\Psi}\left(k v_{0}\right)}{2}+\frac{I_{\Psi}(k v)}{2}$. Since $\Psi(v)$ is strictly convex iff $q_{-}(v)$ is strictly increasing, i.e., $p_{-}(u)$ continuous (see [6]). So $v_{0}=v, \mu$-a.e. $t \in G$. Thus $\|\varphi\|=0$, i.e., $\varphi=0$.

Necessity. Set $f=v+\varphi, \varphi \neq 0$ is a supporting functional of $u$, then

$$
\left.\left.1=\|f\|_{\Psi, q}^{*}=\inf _{k>0} \frac{1}{k}\left(1+\left(\rho^{*}(k f)\right)^{q}\right)^{\frac{1}{q}}\right) \geq \inf _{k>0} \frac{1}{k}\left(1+I_{\Psi}^{q}(k v)\right)^{\frac{1}{q}}\right)=\|v\|_{\Psi, q}
$$

So $u \notin E_{\Phi, p}$, by Lemma 5.2, there exist singular functionals $\varphi_{i},\left\|\varphi_{i}\right\|=1, \varphi_{i}(u)=\theta(u),(i=1,2), \quad$ and $\varphi_{1} \neq \varphi_{2}$. Let $f_{i}=v+\|\varphi\| \cdot \varphi_{i}$. Then $f_{1} \neq f_{2}$ and by Theorem 3.2, $\left\|f_{1}\right\|_{\Psi, q}^{*}=\left\|f_{2}\right\|_{\Psi, q}^{*}=1$. By Theorem 5.3, we have

$$
\begin{aligned}
f_{i}(u) & =\int_{G} u(t) v(t) \mathrm{d} t+\|\varphi\| \cdot \varphi_{i}(u)=\int_{G} u(t) v(t) \mathrm{d} t+\|\varphi\| \theta(u) \\
& \geq \int_{G} u(t) v(t) \mathrm{d} t+\varphi(u)=f(u) .
\end{aligned}
$$

Hence, $f_{1}$ and $f_{2}$ are both supporting functionals of $u$, which shows that $u$ is not a smooth point of $L_{\Phi, p}$.

Theorem 5.6 Let $u \in S\left(L_{\Phi, p}\right)(1 \leq p \leq \infty), u \neq 0$ is a smooth point iff 
(i) $a_{\Psi}=0$,

(ii) $1 \leq p<\infty, I_{\Phi}^{p-1}(k u) \cdot I_{\Psi}\left(p_{-}(k|u|)\right)=1$ or $I_{\Phi}^{p-1}(k u) \cdot I_{\Psi}\left(p_{+}(k|u|)\right)=1$ and $\theta(u)<\frac{1}{k}$

(iii) $\quad p=\infty, \theta(u)<1$ and $G(u)=\left\{t \in G: p_{-}(|u|)<p_{+}(|u|)\right\}$ is a null set.

Proof Necessity.

If (i) is not true, then $a_{\Psi}>0$. Suppose $f=v+\varphi(v \neq 0)$ is a supporting functional of $u$. Take $l \in K_{q}(f)$, there exists $c>0$ such that $l c \leq a_{\Psi}$. Set

$$
\bar{v}= \begin{cases}v, & \text { for } t \in \operatorname{supp}(u) \backslash \operatorname{supp}\left(a_{\Psi}\right), \\ l c, & \text { for } t \in \operatorname{supp}\left(a_{\Psi}\right) .\end{cases}
$$

Hence

$$
\begin{aligned}
\|\bar{v}+\varphi\|_{\Psi, q}^{*} & \leq \frac{1}{l}\left(1+\left(I_{\Psi}(l \bar{v})+l\|\varphi\|\right)^{q}\right)^{\frac{1}{q}} \\
& \leq \frac{1}{l}\left(1+\left(I_{\Psi}(l v)+l\|\varphi\|\right)^{q}\right)^{\frac{1}{q}}=\|v+\varphi\|_{\Psi, q}^{*}=1 .
\end{aligned}
$$

Since $(\bar{v}+\varphi)(u)=(v+\varphi)(u)=\|u\|_{\Phi, p}$, we have $\|\bar{v}+\varphi\|_{\Psi, q}^{*} \geq 1$. So $\bar{v}+\varphi$ is also a supporting functional of $u$. But $\bar{v}+\varphi \neq v+\varphi$, thus $u$ is not a smooth point.

(ii) Suppose $I_{\Phi}^{p-1}(k u) \cdot I_{\Psi}\left(p_{-}(k|u|)\right) \neq 1$. By Theorem 4.4, we have $I_{\Phi}^{p-1}(k u) \cdot I_{\Psi}\left(p_{-}(k|u|)\right)=\alpha<1$. If $\theta(u)<\frac{1}{k}$, then Theorem 5.5 implies that all supporting functionals of $u$ are in $L_{\Psi, q}$. Therefore if $I_{\Phi}^{p-1}(k u) \cdot I_{\Psi}\left(p_{+}(k|u|)\right) \neq 1$, then we must have $I_{\Phi}^{p-1}(k u) \cdot I_{\Psi}\left(p_{+}(k|u|)\right)>1$. This implies that the set

$$
V=\left\{v: p_{-}(k|u(t)|) \leq l v \leq p_{+}(k|u(t)|), I_{\Phi}^{p-1}(k u) I_{\Psi}(l v)=1\right\}
$$

contains infinitely many elements, and by Theorem $4.5(\mathrm{~b})$, every $\frac{v}{\|v\|_{\Psi, q}} \cdot \operatorname{sign} u$ is a supporting functional of $u$, which shows that $u$ is not a smooth point of $L_{\Phi, p}$.

Now, we assume $\theta(u)=\frac{1}{k}$, the supporting functional $f=v+\varphi, \varphi \neq 0$ and $\|\varphi\|=\frac{1-\alpha}{k I_{\Phi}^{p-1}(k u)}$ i.e., $I_{\Phi}^{p-1}(k u)\left(I_{\Psi}\left(p_{-}(k|u|)\right)+k\|\varphi\|\right)=1$. By Lemma 5.2, there exist $\varphi_{1}, \varphi_{2} \in F \quad$ such that $\left\|\varphi_{i}\right\|=1 \quad$ and $\varphi_{i}(u)=\theta(u), \quad(i=1,2)$. Define $f_{i}=p_{-}(k|u|)+\|\varphi\| \cdot \varphi_{i} \quad(i=1,2)$. Then $f_{1} \neq f_{2} \quad$ and by Theorem 3.2, $\left\|f_{1}\right\|_{\Psi, q}^{*}=\left\|f_{2}\right\|_{\Psi, q}^{*}=1$. By the Young Inequality and Lemma 2.6, we have

$$
\begin{aligned}
f_{i}(u) & =\int_{G} u(t) p_{-}(k|u(t)|) \mathrm{d} t+\|\varphi\| \cdot \varphi_{i}(u) \\
& =\frac{1}{k}\left(I_{\Phi}(k u)+I_{\Psi}\left(p_{-}(k|u|)\right)\right)+\|\varphi\| \theta(u) \\
& =\frac{1}{k}\left(I_{\Phi}(k u)+I_{\Psi}\left(p_{-}(k|u|)\right)+\|\varphi\|\right) \\
& =\frac{1}{k} s_{p}\left(I_{\Phi}(k u)\right) \cdot s_{q}\left(I_{\Psi}\left(p_{-}(k|u|)\right)+\|\varphi\|\right) \\
& \geq\|u\|_{\Phi, p} \cdot\left\|f_{i}\right\|_{\Psi, q}^{*} .
\end{aligned}
$$


Hence, $f_{1}$ and $f_{2}$ are both supporting functionals of $u$, which shows that $u$ is not a smooth point of $L_{\Phi, p}$.

The paper [6] has given the proof of the necessity of condition (iii).

Sufficiency.

Let $f=v+\varphi$ be a supporting functional of $u$, where $v \in L_{\Psi, q}, \varphi \in F$.

If $1 \leq p<\infty$. Then Theorem $4.4(\mathrm{~b}, \mathrm{c})$ shows that $p_{-}(k|u|) \leq l|v| \leq p_{+}(k|u|)$, where $k \in K_{p}(u), l \in K_{q}(f) \quad$ and $\quad l=\frac{1}{\|f\|_{\psi_{\infty}}^{*}}, \quad$ if $\quad q=\infty$. Hence if $I_{\Phi}^{p-1}(k u) \cdot I_{\Psi}\left(p_{-}(k|u|)\right)=1$ holds, then by Theorem 4.4(b, c) we deduce that $\varphi=0$ and $v=\frac{p_{-}(k|u|)}{\left\|p_{-}(k|u|)\right\|_{\Psi, q}} \cdot \operatorname{sign} u$ is the unique supporting functional of $u$. If $I_{\Phi}^{p-1}(k u) \cdot I_{\Psi}\left(p_{+}(k|u|)\right)=1$ holds, by Theorem $4.4(\mathrm{~b}, \mathrm{c})$, we have $\varphi=0$. Thus $v=\frac{p_{+}(k|u|)}{\left\|p_{+}(k|u|)\right\|_{\Psi, q}} \cdot \operatorname{sign} u$ the unique supporting functional of $u$.

When $p=\infty$. Theorem 4.4(a) and Lemma 2.9 imply that all supporting functional of $u$ are contained in $L_{\Psi, 1}$. By Theorem 11 , we know $v=\frac{p_{-}(u)}{\left\|p_{-}(u)\right\|_{\Psi, 1}} \cdot \operatorname{sign} u$ is the unique supporting functional at $u$.

Theorem $5.7 L_{\Phi, p}(1 \leq p \leq \infty)$ is smooth if and only if:

(i) $a_{\Psi}=0$;

(ii) $p_{-}(u)$ is continuous;

(iii) $\Phi \in \Delta_{2}(\infty)$.

Proof Sufficiency.

The condition (iii) implies $L_{\Phi, p}=E_{\Phi, p}$. For any $u \in E_{\Phi, p}$, we have $\theta(u)=0<\frac{1}{k}$. If condition (ii) holds, then for every $u \in S\left(L_{\Phi, p}\right), p_{-}(u)=p_{+}(u)$, i.e., $V$ has only one function where $V$ defined as in Theorem 5.6. By condition (i) and Theorems 5.5 and 5.6, $u$ is a smooth point of $E_{\Phi, p}$.

Necessity.

The condition (i) follows from Theorem 5.6.

(ii) If $p_{-}(u)$ is not continuous, then there exist $A, v_{1}, v_{2}$ such that $q_{-}(v)=A$ for all $v \in\left[v_{1}, v_{2}\right]$. We can find $G_{1} \subset G$ such that

$$
\left(\Phi(A) \mu\left(G_{1}\right)\right)^{p-1} \cdot \Psi\left(p_{-}(A)\right) \mu\left(G_{1}\right)<1 .
$$

Select $a>0$ such that

$$
\left(\Phi(A) \mu\left(G_{1}\right)+\Phi\left(q_{-}(a)\right) \mu\left(G \backslash G_{1}\right)\right)^{p-1}\left(\Psi\left(p_{-}(A)\right) \mu\left(G_{1}\right)+\Psi(a) \mu\left(G \backslash G_{1}\right)\right)>1 .
$$

There exists $G_{2} \subset G \backslash G_{1}$, satisfying

$$
\left(\Phi(A) \mu\left(G_{1}\right)+\Phi\left(q_{-}(a)\right) \mu\left(G_{2}\right)\right)^{p-1}\left(\Psi\left(p_{-}(A)\right) \mu\left(G_{1}\right)+\Psi(a) \mu\left(G_{2}\right)\right)=1 .
$$

Set $u(t)=A \chi_{G_{1}}(t)+q_{-}(a) \chi_{G_{2}}(t)$, then $I_{\Phi}^{p-1}(u) \cdot I_{\Psi}\left(p_{-}(|u|)\right)=1$. Divide the set $G_{1}$ into two sets $E$ and $F$, with $\mu E=\mu F$ and let

$$
w_{1}(t)=v_{1} \chi_{E}(t)+v_{2} \chi_{F}(t)+a \chi_{G_{2}}(t),
$$




$$
w_{2}(t)=v_{2} \chi_{E}(t)+v_{1} \chi_{F}(t)+a \chi_{G_{2}}(t),
$$

then $q_{-}\left(w_{i}(t)\right)=A \chi_{G_{1}}(t)+q_{-}(a) \chi_{G_{2}}(t)=u(t)(i=1,2)$.

Let $p=1$. Then $I_{\Psi}\left(p_{-}(u)\right)=I_{\Psi}\left(w_{i}\right)=1$ and $\left\|w_{i}\right\|_{\Psi, \infty}=1(i=1,2)$.

$$
\|u\|_{\Phi, 1}=\|u\|_{\Phi}^{o}=\int_{G} u(t) p_{-}(u(t)) \mathrm{d} t=\int_{G} u(t) w_{i}(t) \mathrm{d} t .
$$

Hence, $w_{1}$ and $w_{2}$ are both supporting functionals of $\frac{u}{\|u\|_{\Phi, 1}}$. Thus, $\frac{u}{\|u\|_{\Phi, 1}}$ is not a smooth point of $L_{\Phi, 1}$.

Let $1<p<\infty$ and $v_{i}=\frac{w_{i}}{\left\|w_{i}\right\|_{\Psi, q}}$, by the Young inequality and Lemma 2.6, we have

$$
\begin{aligned}
1 & \geq\left\langle v_{i}, \frac{u}{\|u\|_{\Phi, p}}\right\rangle=\left\langle\frac{w_{i}}{\left\|w_{i}\right\|_{\Psi, q}}, \frac{u}{\|u\|_{\Phi, p}}\right\rangle \\
& =\frac{1}{\left\|w_{i}\right\|_{\Psi, q}} \cdot \frac{1}{\|u\|_{\Phi, p}} \int_{G} u(t) w_{i}(t) \mathrm{d} t \\
& =\frac{1}{\left\|w_{i}\right\|_{\Psi, q}} \cdot \frac{1}{\|u\|_{\Phi, p}}\left(I_{\Phi}(u)+I_{\Psi}\left(w_{i}\right)\right) \\
& =\frac{1}{\left\|w_{i}\right\|_{\Psi, q}} \cdot \frac{1}{\|u\|_{\Phi, p}} s_{p}\left(I_{\Phi}(u)\right) \cdot s_{q}\left(I_{\Psi}\left(w_{i}\right)\right) \geq 1 .
\end{aligned}
$$

Hence, $v_{i}=\frac{w_{i}}{\left\|w_{i}\right\|_{\Psi, q}}(i=1,2)$ is a supporting functional of $\frac{u}{\|u\|_{\Phi, p}}$, which implies $\frac{u}{\|u\|_{\Phi, p}}$ is not a smooth point of $L_{\Phi, p}$.

Let $p=\infty$. In an analogous way as above, we can construct

$$
u(t)=A \chi_{G_{1}}(t)+q_{-}(a) \chi_{G_{2}}(t)
$$

such that $\Phi(A) \mu\left(G_{1}\right)+\Phi\left(q_{-}(a)\right) \mu\left(G_{2}\right)=1$. We define $w_{1}$ and $w_{2}$ as (7) and (8). Then $u \in E_{\Phi, \infty},\|u\|_{\Phi, \infty}=1$ and $I_{\Phi}(u)=I_{\Phi}\left(q_{-}\left(w_{i}\right)\right)=1$. Hence

$$
1=\left\|\frac{w_{i}}{\left\|w_{i}\right\|_{\Psi, 1}}\right\|_{\Psi, 1}=\int_{G} \frac{w_{i}(t)}{\left\|w_{i}\right\|_{\Psi, 1}} q_{-}\left(w_{i}(t)\right) \mathrm{d} t=\int_{G} \frac{w_{i}(t)}{\left\|w_{i}\right\|_{\Psi, 1}} u(t) \mathrm{d} t .
$$

Hence, $v_{i}=\frac{w_{i}}{\left\|w_{i}\right\|_{\Psi, 1}}(i=1,2)$ is a supporting functional of $u$, which implies $u$ is not a smooth point of $L_{\Phi, \infty}$.

(iii) Let $1 \leq p<\infty$. We assume that $\Phi \notin \Delta_{2}(\infty)$. By the definition of $\Phi \notin \Delta_{2}(\infty)$, there exist $u_{n} \nearrow \infty$ such that $\Phi\left(\left(1+\frac{1}{n}\right) u_{n}\right)>n \cdot 2^{n+1} \Phi\left(u_{n}\right)$ where $n \in N$ (see [6]). Observing that

$$
\Phi\left(\left(1+\frac{1}{n}\right) u_{n}\right)=\int_{0}^{\left(1+\frac{1}{n}\right) u_{n}} p_{-}(t) \mathrm{d} t \quad\left(u_{n}>0\right),
$$

and 


$$
\Phi\left(u_{n}\right) \geq \int_{\left(1-\frac{1}{n}\right) u_{n}}^{u_{n}} p(t) \mathrm{d} t>\frac{1}{n} u_{n} p_{-}\left(\left(1-\frac{1}{n}\right) u_{n}\right) \quad\left(u_{n}>0\right),
$$

we have

$$
\begin{aligned}
& \left(1+\frac{1}{n}\right) u_{n} p_{-}\left(\left(1+\frac{1}{n}\right) u_{n}\right) \geq \Phi\left(\left(1+\frac{1}{n}\right) u_{n}\right)>n \cdot 2^{n+1} \Phi\left(u_{n}\right) \\
& \quad>2^{n+1} u_{n} p_{-}\left(\left(1-\frac{1}{n}\right) u_{n}\right)
\end{aligned}
$$

Therefore $p_{-}\left(\left(1+\frac{1}{n}\right) u_{n}\right)>2^{n} p_{-}\left(\left(1-\frac{1}{n}\right) u_{n}\right)$. Without loss of generality, we assume $\frac{u_{2}}{2} \cdot p_{-}\left(\frac{u_{2}}{2}\right) \mu \stackrel{n}{G}>1$, then there exist disjoint $\left\{G_{n}\right\}(n \geq 3)$ in $\sum$ such that $\left(1-\frac{1}{n}\right) u_{n} p_{-}\left(\left(1-\frac{1}{2}\right) u_{n}\right) \mu G_{n}=\frac{1}{2^{n}}, \Phi\left(u_{n}\right) \mu G_{n}=\frac{1}{2^{n+1}}$.

Define $x=\sum_{n=3}^{\infty^{n}}\left(1-\frac{1}{n}\right) u_{n} \chi_{G_{n}}^{2^{n}}$, then

$$
\begin{aligned}
& I_{\Phi}(x)+I_{\Psi}\left(p_{-}(x)\right)=\int_{G} x(t) p_{-}(x(t)) \mathrm{d} t \\
& =\sum_{n=3}^{\infty}\left(1-\frac{1}{n}\right) u_{n} p_{-}\left(\left(1-\frac{1}{n}\right) u_{n}\right) \mu G_{n}=\sum_{n=3}^{\infty} \frac{1}{2^{n}}<1 .
\end{aligned}
$$

We imply $I_{\Phi}(x)<1$ and $I_{\Psi}\left(p_{-}(x)\right)<1$. Thus, we have $I_{\Phi}^{p-1}(x) I_{\Psi}\left(p_{-}(x)\right)<1$.

For any $l>1$, let $m>2$ satisfy $\left(1-\frac{1}{m}\right) l>1+\frac{1}{n}$. Then

$$
\begin{aligned}
I_{\Phi}(x)+I_{\Psi}\left(p_{-}(l x)\right) & \geq \int_{G} x(t) p_{-}(l x(t)) \mathrm{d} t \\
& \geq \sum_{n>m}^{\infty}\left(1-\frac{1}{n}\right) u_{n} p_{-}\left(\left(1+\frac{1}{n}\right) u_{n}\right) \mu G_{n} \\
& \geq \sum_{n>m}^{\infty}\left(1-\frac{1}{n}\right) u_{n} \cdot 2^{n} p_{-}\left(\left(1-\frac{1}{n}\right) u_{n}\right) \mu G_{n}=\infty .
\end{aligned}
$$

This shows $I_{\Psi}\left(p_{-}(l x)\right)=\infty$. So we have $I_{\Phi}^{p-1}(l x) I_{\Psi}\left(p_{-}(l x)\right)=\infty$.

$$
I_{\Phi}(l x)>\sum_{n>m}^{\infty} \Phi\left(\left(1+\frac{1}{n}\right) u_{n}\right) \mu G_{n}=\sum_{n>m}^{\infty} n \cdot 2^{n+1} \Phi\left(u_{n}\right) \mu G_{n}=\infty .
$$

We imply $\theta(x)=1$ and $K_{p}(x)=\{1\}$. By Theorems 5.5 and 5.6, $x$ is not a smooth point of $L_{\Phi, p}$.

Let $p=\infty$. Then $[4,31]$ have given the proof of sufficiency in different ways. So we omit it here.

Acknowledgements This work was supported by the Natural Science Foundation of China (11871181), (11701125).

Open Access This article is licensed under a Creative Commons Attribution 4.0 International License, which permits use, sharing, adaptation, distribution and reproduction in any medium or format, as long as you give appropriate credit to the original author(s) and the source, provide a link to the Creative 
Commons licence, and indicate if changes were made. The images or other third party material in this article are included in the article's Creative Commons licence, unless indicated otherwise in a credit line to the material. If material is not included in the article's Creative Commons licence and your intended use is not permitted by statutory regulation or exceeds the permitted use, you will need to obtain permission directly from the copyright holder. To view a copy of this licence, visit http://creativecommons.org/ licenses/by/4.0/.

\section{References}

1. Aliprantis, C.D., Burkinshaw, O.: Positive Operators. Springer (1985)

2. Ando, T.: Linear functionals on Orlicz spaces. Nieuw Arch. Wiskd. 8(8), 1-16 (1960)

3. Chen, L., Cui, Y.: Complex extreme points and complex rotundity in Orlicz function spaces equipped with the p-Amemiya norm. Nonlinear Anal. 73(5), 1389-1393 (2010)

4. Chen, S.: Smoothness of Orlicz spaces. Comment. Math. XXVII, 49-58 (1987)

5. Chen, S.: The smooth point of Orlicz spaces. Nat. Sci. J. Harbin Norm. Univ. 5, 1-4 (1989)

6. Chen, S.: Geometry of Orlicz spaces. Diss. Math. 365 (1996)

7. Chen, S., Hudzik, H., Kaminska, A.: Support functionals and smooth points in Orlicz function spaces equipped with the Orlicz norm. Math. Jpn. 39(2), 271-279 (1994)

8. Cui, Y., Hudzik, H., Wisla, M., Wlazlak, K.: Non-squareness properties of Orlicz spaces equipped with the p-Amemiya norm. Nonlinear Anal. Theory Methods Appl. 75(10), 3973-3993 (2012)

9. Cui, Y., Duan, L., Hudzik, H., Wisla, M.: Basic theory of p-Amemiya norm in Orlicz spaces: extreme points and rotundity in Orlicz spaces endowed with these norms. Nonlinear Anal. 69(5-6), 1796-1816 (2008)

10. Cui, Y., Hudzik, H., Li, J.: Some fundamental properties for duals of Orlicz spaces. Nonlinear Anal. Theory Methods Appl. 73(8), 2353-2360 (2010)

11. Cui, Y., Hudzik, H., Li, J., Wisla, M.: Strongly extreme points in Orlicz spaces equipped with the p-Amemiya norm. Nonlinear Anal. 71, 6343-6364 (2009)

12. Cui, Y., Hudzik, H., Wisla, M.: Monotonicity properties and dominated best approximation problems in Orlicz spaces equipped with the p-Amemiya norm. J. Math. Anal. Appl. 432(2), 1095-1105 (2015)

13. Grzaślewicz, R., Hudzik, H.: Smooth points of Orlicz spaces equipped with Luxemburg norm. Math. Nachr. 155(1), 31-45 (1992)

14. He, X., Cui, Y., Hudzik, H.: The fixed point property of Orlicz sequence spaces equipped with the p-Amemiya norm. Fixed Point Theory Appl. 2013(1), 1-18 (2013)

15. Hudzik, H., Maligranda, L.: Amemiya norm equals Orlicz norm in general. Indag. Math. 11(4), 573-585 (2000)

16. Hudzik, H., Zbaszyniak, Z.: Smoothness in Musielak-Orlicz spaces equipped with Orlicz norm. Collect. Math. 48(8-9), 543-561 (1997)

17. Kaczmarek, R.: Uniform rotundity of Orlicz function spaces equipped with the p-Amemiya norm. Math. Nachr. 291(10), 1514-1532 (2018)

18. Krasnoselskii, M.A., Rutickii, Y.B.: Convex Functions and Orlicz Spaces. P. Noordhoff Ltd (1961)

19. Li, X., Cui, Y.: The dual space of Orlicz space equipped with p-Amemiya norm. J. Harbin Univ. Sci. Technol. 16(1), 110-112 (2011)

20. Luxemburg, W.A.J., Zaanen, A.C.: Conjugate spaces of Orlicz spaces. Indag. Math. 59, 217-228 (1956)

21. Musielak, J.: Orlicz Spaces and Modular Spaces. Springer (1983)

22. Musielak, J., Orlicz, W.: On modular spaces. Stud. Math. 18(1), 1-128 (1959)

23. Nakano, H.: Topology and Linear Topological Spaces. Maruzen Co.Ltd (1951)

24. Orlicz, W.: A note on modular spaces. Bull. Acad. Pol. Sci. Math. Astron. Phys. 9, 157-162 (1961)

25. Rao, M.M., Ren, Z.: Theory of Orlicz Spaces. Marcek Dekker Inc (1991)

26. Shi, Z., Wang, T.: On the smoothness of Orlicz sequence spaces equipped with Orlicz norm. Collect. Math. 47(2), 105-109 (1996)

27. Vigelis, R.F., Cavalcante, C.C.: Smoothness of the Orlicz norm in Musielak-Orlicz function spaces. Math. Nachr. 287(8-9), 1025-1041 (2014) 
28. Wang, B., Zhang, Y.: The smooth points of Orlicz sequence spaces. Nat. Sci. J. Harbin Norm. Univ. 7(3), 18-22 (1991)

29. Wisla, M.: Geometric properties of Orlicz spaces equipped with the p-Amemiya norms-results and open questions. Comment. Math. 55, 183-209 (2015)

30. Wisla, M.: Orlicz spaces equipped with the s-norms. J. Math. Anal. Appl. 483(2), 1-30 (2020)

31. Wu, C., Wang, T., Chen, S., Wang, Y.: Geometric Theory of Orlicz Spaces. Print House (1986) 